\title{
A Systematic Review and Meta-Analysis of Human Milk Feeding and Short-Term Growth in Preterm and Very Low Birth Weight Infants
}

\author{
Machiko Suganuma ${ }^{1,+}$, Alice R. Rumbold ${ }^{1,2,+}$, Jacqueline Miller ${ }^{1,3} \mathbb{D}^{\text {, Yan Fong Chong }}{ }^{3}$ \\ and Carmel T. Collins $1,2, *$ \\ 1 SAHMRI Women and Kids, South Australian Health and Medical Research Institute, \\ Adelaide, SA 5006, Australia; sakai197723@yahoo.co.jp (M.S.); alice.rumbold@sahmri.com (A.R.R.); \\ jacqueline.miller@sahmri.com (J.M.) \\ 2 Adelaide Medical School, The University of Adelaide, Adelaide, SA 5006, Australia \\ 3 College of Nursing and Health Sciences, Flinders University, Adelaide, SA 5001, Australia; \\ chong.yanfong@gmail.com \\ * Correspondence: carmel.collins@sahmri.com \\ + Both authors contributed equally to this work.
}

Citation: Suganuma, M.; Rumbold, A.R.; Miller, J.; Chong, Y.F.; Collins,

C.T. A Systematic Review and

Meta-Analysis of Human Milk Feeding and Short-Term Growth in Preterm and Very Low Birth Weight Infants. Nutrients 2021, 13, 2089.

https://doi.org/10.3390/nu13062089

Academic Editor: Nadja Haiden

Received: 10 May 2021

Accepted: 10 June 2021

Published: 18 June 2021

Publisher's Note: MDPI stays neutral with regard to jurisdictional claims in published maps and institutional affiliations.

Copyright: (c) 2021 by the authors. Licensee MDPI, Basel, Switzerland. This article is an open access article distributed under the terms and conditions of the Creative Commons Attribution (CC BY) license (https:/ / creativecommons.org/licenses/by/ $4.0 /)$.

\begin{abstract}
Human milk (HM) is the gold standard for feeding infants but has been associated with slower growth in preterm infants compared with preterm formula. This systematic review and meta-analysis summarises the post-1990 literature to examine the effect of HM feeding on growth during the neonatal admission of preterm infants with birth weight $\leq 1500 \mathrm{~g}$ and/or born $\leq 28$ weeks' gestation. Medline, PubMed, CINAHL, and Scopus were searched, and comparisons were grouped as exclusive human milk (EHM) vs. exclusive preterm formula (EPTF), any HM vs. EPTF, and higher vs. lower doses of HM. We selected studies that used fortified HM and compared that with a PTF; studies comparing unfortified HM and term formula were excluded. Experimental and observational studies were pooled separately. The GRADE system was used to evaluate risk of bias and certainty of evidence. Forty-four studies were included with 37 ( $n=9963$ infants) included in the meta-analyses. In general, due to poor quality studies, evidence of the effect of any HM feeds or higher versus lower doses of HM was inconclusive. There was a possible effect that lower doses of HM compared with higher doses of HM improved weight gain during the hospital admission, and separately, a possible effect of increased head circumference growth in infants fed EPTF vs. any HM. The clinical significance of this is unclear. There was insufficient evidence to determine the effects of an exclusive HM diet on any outcomes.
\end{abstract}

Keywords: preterm infant; human milk; growth

\section{Introduction}

Mother's own milk (MOM) is the feed of choice for preterm infants [1] because of clear advantages for immunological, gastrointestinal, and neurodevelopmental health and function [2,3]. Where there is insufficient MOM, current recommendations are to use appropriately screened and pasteurised donor human milk (DHM) if available, and then preterm formula (PTF) [2,4]. However, human milk (HM) alone is insufficient to support the growth requirements of preterm and very low birth weight $(<1500 \mathrm{~g}$, VLBW) infants, with many early studies reporting poorer growth in infants fed unfortified HM than infants fed PTF [5]. Hence, nutrient fortification of HM is now standard clinical practice for VLBW babies in many settings [6]. Even with routine fortification of HM, there is inconsistent evidence about the impact of HM feeds on infant growth. For example, some studies have reported slower weight, length, and head circumference (HC) gain, whereas others suggest HM (MOM/DHM) adequately supports early postnatal growth compared with formula feeding [7-9]. A recent Cochrane systematic review failed to identify any randomised 
trials that specifically examined HM feeds versus preterm formula in preterm or low birth weight infants [10]. In another Cochrane review of the same population comparing formula versus DHM, greater short-term growth was shown in infants fed formula, even where DHM had been fortified [11]. However, there is also evidence that HM supports better quality growth, as formula-fed preterm infants have increased fat mass at term corrected age when compared to HM-fed infants [12].

Given the conflicting evidence, a greater understanding of how HM feeding, whether with MOM or DHM, affects growth in preterm and VLBW infants is necessary. This is particularly important as accumulated growth deficits in the neonatal period are associated with unfavourable neurodevelopmental outcomes in later life [13]. This review builds on our previous review of human milk feeding and morbidity in VLBW infants [3] and aims to provide a direct comparison of growth between infants fed exclusive PTF (EPTF) and exclusive HM (EHM) and to explore the associations with various doses of HM intake and growth. We summarise the evidence on growth, i.e., weight, length, and HC gain, and body composition (proportion of fat mass and fat-free mass). The present review provides a comprehensive overview inclusive of both randomised controlled trials (RCTs) and, given the limited number of trials, observational studies, and varying doses of $\mathrm{HM}$ feeding, reflective of current practice for VLBW infants.

\section{Materials and Methods}

\subsection{Registration and Reporting}

This review is registered with PROSPERO International prospective register of systematic reviews (PROSPERO 2018 CRD42018104389) and the protocol is available from http:/ / www.crd.york.ac.uk/PROSPERO/display_record.php?ID=CRD42018104389 (accessed on 16 June 2021). The Preferred Reporting Items for Systematic Reviews and Meta-Analyses (PRSMA) [14] statement has been followed.

\subsection{Eligibility Criteria}

\subsubsection{Types of Studies}

RCTs and observational studies, published from 1990 onwards, were considered for inclusion in this review. All component studies of relevant systematic reviews were also considered. Secondary analyses of RCTs were considered as cohort studies. Comparisons between groups in RCTs that included a non-randomised arm, usually a reference feeding group, were considered as a cohort design (e.g., Costa-Orvay, 2011 [15]).

\subsubsection{Types of Participants}

Infants born at $\leq 28$ weeks' gestation and/or study populations with a reported mean birth weight $\leq 1500 \mathrm{~g}$ were considered for inclusion. Post-discharge studies were excluded. As quantifying the exposure of HM was necessary for group studies, we excluded studies where this could not be done reliably, e.g., where feeding intake data were recalled retrospectively or measured at only one time point and extrapolated for the neonatal admission.

\subsubsection{Types of Intervention}

Studies comparing the effects of HM were grouped according to the following exposure categories:

EHM compared with EPTF. This provides a direct evaluation of the effect of the two opposite feeding methods.

Any HM (includes EHM or HM plus PTF) compared with EPTF. This explores the effect of any HM when infants also receive PTF.

Higher-dose HM (includes EHM plus high dose of HM with PTF) was compared with low-dose HM (lower dose of HM with some PTF). This comparison was included to explore the dose-related effect of HM. No a priori categories were defined for a 'higher' or 'lower' dose of HM. Therefore, studies included here may have compared EHM with mixed feeding groups, or alternatively, all infant groups may have been mixed fed, with 
those having a higher proportion of enteral intake as HM compared with those having a lower proportion of enteral intake as HM.

As this review is intended to reflect contemporary feeding practices, we selected studies that used fortified HM and compared that with a PTF. Studies comparing unfortified $\mathrm{HM}$ and term formula were excluded.

\subsubsection{Type of Outcome Measure}

Outcome measures included:

In-hospital growth (weight, length, and HC gains), where growth rates during the neonatal admission were measured. Where findings were expressed in similar units (i.e., $\mathrm{g} / \mathrm{kg} / \mathrm{d}, \mathrm{g} / \mathrm{d}, \mathrm{cm} / \mathrm{wk}, \mathrm{z}$-scores), they were combined in meta-analyses.

In-hospital body composition (fat mass, lean mass, grams, and the relative percentages). Results at the study's end were combined in meta-analyses.

In-hospital growth (i.e., during the neonatal admission) was chosen as the most appropriate period to study, as it is commonly reported and has previously been identified as a sensitive period influencing later neurodevelopmental outcomes [16]. Considerable variation in reporting infant growth has been described [17], particularly regarding weight gain, which is variously reported in $\mathrm{g} / \mathrm{kg} / \mathrm{d}, \mathrm{g} / \mathrm{d}$, or z-score. There is also a lack of standardisation over the measurement period including birth to discharge or some other common start or endpoint such as from when birth weight is regained or 36 weeks post menstrual age. We report growth velocity and z-score change to show growth status changes over time [17]. Body composition measures also vary considerably in the measurement tools used, i.e., DEXA, air displacement plethysmography, and bioelectrical impedance. We have taken a broad and inclusive approach to these variations in an attempt to identify as much literature as possible. Measurement details have been documented, only data with similar units have been combined in meta-analyses, and where results for two different time points have been reported, the time point that is closest to the other studies included has been used for the meta-analyses.

\subsection{Information Sources and Study Selection}

Searches were conducted in June 2020 in the following databases: Medline (Ovid), PubMed, Scopus, The Cochrane Central Register of Controlled Trials (Wiley), and CINAHL (EBSCOhost). Results were restricted to English language published from 1990.

Search terms for each key concept (preterm infant, HM feeding, weight/length/HC gain) included database-specific broad subject terms, e.g., CINAHL Headings in CINAHL and MeSH in Cochrane Library and PubMed, and a wide range of synonyms and free-text terms were searched as text words. Necrotising enterocolitis was included as a search keyword to include studies that may report growth as a secondary outcome. Reference lists of articles were hand-searched to identify further relevant articles. Citations were exported to Covidence [18] for organisation and screening. Two authors independently screened articles against the eligibility criteria for inclusion. Disagreements were resolved by discussion between the two authors, with a third author involved if necessary.

\subsection{Data Extraction, Risk of Bias in Individual Studies, and Data Synthesis}

Two authors extracted the relevant data into tables. Data extracted were limited to comparisons typical of standard clinical care, e.g., feeding PTF with HM fortified with a multicomponent fortifier. Therefore, arms of studies where an experimental formula was used (e.g., with the addition of long-chain polyunsaturated fatty acids as in Fewtrell 2002 [19] and $\mathrm{O}^{\prime}$ Connor 2001 [20]) were excluded, as were arms of studies using HM fortified with minerals alone [21]. In addition, the data extracted were aligned with our gestational age and birth-weight criteria. If a study included a wider gestational age and birth-weight range but reported relevant data as a subgroup that met our criteria, such as Lok 2017 [22], we extracted only those data. 
The risk of bias was assessed using the Cochrane Risk of Bias Tool [23] for RCTs and the Academy of Nutrition and Dietetics Quality Criteria Checklist [24] for other study designs. RCTs reporting on a subset of data not based on randomised status were assessed for study quality as a cohort design.

Where meta-analyses were possible, results of RCTs and observational studies were included as separate subgroups, using Review Manager (RevMan), Version 5.4.1 [25], with data expressed as mean difference (MD) with $95 \%$ confidence interval (CI). Where only medians with a measure of dispersion were available, these were converted to means and standard deviations (SD) using an online calculator (available at: http:/ / www. math.hkbu.edu.hk/ tongt/papers/median2mean.html, accessed on 21 June 2021) [26,27]. Where mean and 95\% CI were reported, these were converted to SD [23], and where groups were combined for meta-analyses, Cochrane methods were followed [23]. A random effects model was used where statistical heterogeneity was significant $\left(\mathrm{I}^{2}>50 \%\right)$. Where studies have two or more intervention groups that fall into the designated categories, we report the combined means as calculated in Revman [25]. We have attempted to avoid including the same group of infants twice in the same meta-analysis, while striving to optimise precision by including relevant groups. Where it has not been possible to tease out the overlapping infants, we have not adjusted the $n$ value but noted this in text, e.g., Huston 2018 [28], which includes some infants also reported in Huston 2014 [29], and noted if inclusion of this study changed the outcomes.

A 'Summary of Findings' table was prepared for each comparison using the GRADE system (GRADEpro GDT, 2015) [30]. RCTs with no limitations are considered high-quality evidence and observational studies as providing low-quality evidence. Studies can then be downgraded by one (for serious concern) or two (for very serious concerns) based on risk of bias, inconsistency, indirectness, imprecision, and publication bias. For each outcome, we report our certainty in the findings as very low, low, moderate, or high separately, according to study design (RCTs, observational).

To interpret the overall evidence for each outcome and comparison, we used the following terminology:

Clear effect/clear evidence of no effect: The certainty of evidence is moderate or above with a clinically important result from RCTs, ideally aligning with results from observational studies or moderate certainty evidence from observational studies and with reasonable numbers of events and/or participants.

Probably an effect/probably no effect: There is moderate certainty from either RCTs or observational studies, and point estimates may be different between the two study types with overlapping CIs but can be explained (e.g., through heterogeneity). There are large numbers of participants and studies.

Possible effect/possibly no effect: There is low/ moderate certainty with CIs, which may suggest a difference, although not reaching conventional statistical significance, or with a confidence interval, which indicates a trivial difference only.

Inconclusive: The certainty of evidence is very low to low, CIs are wide, and the number of participants and studies is low.

Where possible, the overall effect size (MD, 95\% CI) has been reported. Figures showing forest plots for all outcomes are presented in the manuscript. For visual comparison, we have displayed a forest plot for comparisons that have only one study; however, we have computed a GRADE certainty rating only where there are two or more studies.

\section{Results}

The search and selection processes are presented in Supplementary Materials (Figure S1). Forty-four papers were identified for this review and included nine RCTs [15,19-21,31-35], one non-randomised intervention trial [36], three secondary analyses of RCTs [9,37,38], six interrupted time series studies [8,28,39-42], and 25 cohort studies [7,22,29,43-64]. Thirtyseven studies could be included in the meta-analyses [7-9,15,19-22,28,29,31-37,39,40,42,44, 47-52,54-61,63,64]. 
For the four RCTs, we could directly compare data from randomised arms [31-34]. In five RCTs, we compared data to a non-randomised reference group, and consequently, these were assessed for study quality and treated as non-randomised trials [15,19-21,35]. Notably, for the RCT by Schanler 2005 [33], we also made comparisons between a randomised group and a non-randomised reference arm.

Table 1 provides details of the included studies. Individual summaries of findings tables for each comparison and outcome are presented in Supplementary Materials (Tables S2-S12), and a collated summary of findings table for all outcomes is presented in Table 2. Results for studies not included in a meta-analysis have been tabulated in Supplementary Materials (Table S1).

\subsection{Risk of Bias and General Characteristics}

Two of the RCTs [32,33] were rated as low risk of bias, one was assessed as moderate risk due to unclear sequence generation and allocation concealment [31], and another was considered high risk due to non-blinding of outcome assessors [34].

Of the non-randomised and observational studies, 28 were assessed as low risk of bias $[7-9,15,19-22,35,38-43,45,47,49,50,52,55,57-61,63,64], 11$ as moderate $[28,29,36,37,44$, 46,48,51,54,56,62], and 1 as high [53] (Table 1).

There was some clinical heterogeneity in the time period over which growth was calculated, with starting points including birth, when birth weight was regained, weight nadir, attainment of $50 \%$ oral feeds, and study start; and endpoints including discharge, term corrected age, and study end (Table 1).

\subsection{Weight Gain and Human Milk Feeding}

Weight gain was reported in 41 studies [7-9,19-22,28,29,31-36,38-53,55-64]. Twentyfour studies reported $\mathrm{g} / \mathrm{kg} / \mathrm{d}$, with 20 included in the meta-analysis $[9,19-21,28,29,33-$ $35,39,40,47,50,52,57-61,64]$ and four unable to be included [43,45,56,62]. Four studies reported weight gain in $\mathrm{g} / \mathrm{d}[31,36,48,53]$, with three included in the meta-analysis $[31,36,48]$. Nineteen studies reported weight gain as change in z-scores with 14 included in metaanalyses $[7,8,22,28,32,42,44,47,49-51,55,61,63]$ and five not included $[38,41,43,46,62]$.

\subsubsection{Comparison 1: Exclusive Preterm Formula vs. Exclusive Human Milk}

Randomised Trials: One small RCT [31] showed no difference in weight gain (g/d) between EPTF and EHM groups (MD 2.00, 95\% CI -1.54 to 5.54, $n=53$, Figure 1, moderate risk of bias, Table S2).

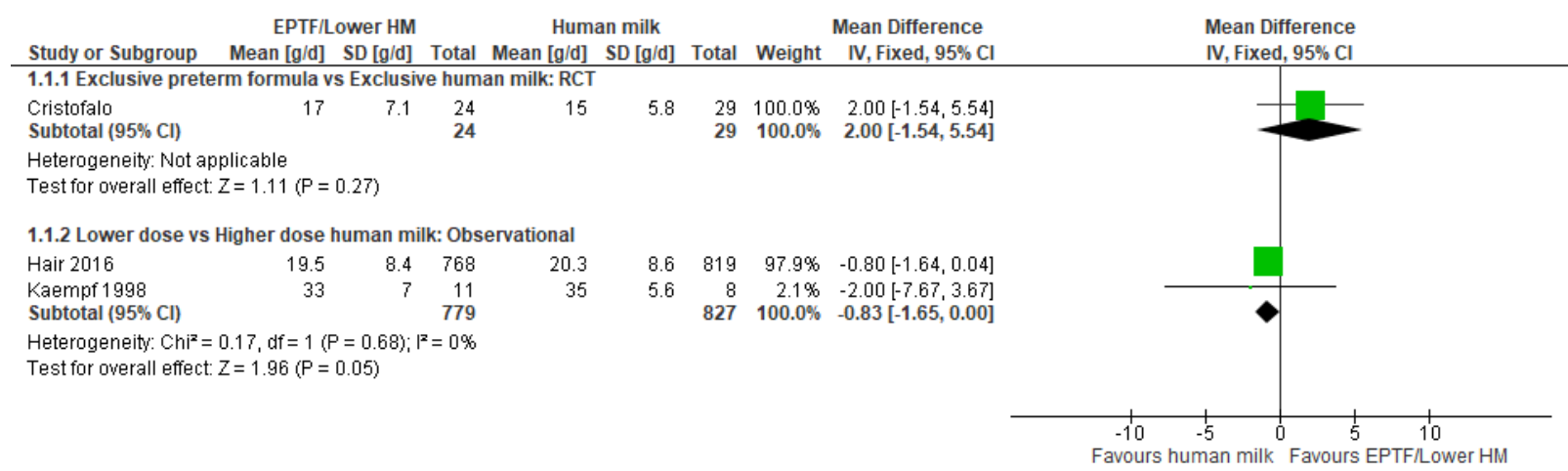

Figure 1. Forest plot of mean difference in weight gain $(\mathrm{g} / \mathrm{d})$ and human milk intake. 
Table 1. Characteristics of included studies.

\begin{tabular}{|c|c|c|c|c|c|c|c|}
\hline $\begin{array}{l}\text { Study } \\
\text { Country }\end{array}$ & Design & $\begin{array}{l}\text { Participants } \\
\text { BW, g; GA, wk; } n\end{array}$ & $\begin{array}{l}\text { Intervention } \\
\text { (Proportion of HM \% } \\
\text { Unless Stated Elsewhere) }\end{array}$ & $\begin{array}{l}\text { Comparisons for This } \\
\text { Review } \\
\text { C1: EHM vs. EPTF } \\
\text { C2: Any HM vs. EPTF } \\
\text { C3: High- vs. Low-Dose HM }\end{array}$ & Study Duration & Outcomes & Risk of Bias \\
\hline \multicolumn{8}{|c|}{ Randomised Controlled Trials } \\
\hline $\begin{array}{l}\text { Cristofalo (2013) [31] } \\
\text { USA and Australia }\end{array}$ & $\mathrm{RCT}$ & $\begin{array}{l}53 \text { infants } \\
\text { Gp1: } 996 \pm 152 ; 27.7 \pm 1.5 ; 29 \\
\text { Gp2: } 983 \pm 207 ; 27.5 \pm 2.4 ; 24\end{array}$ & $\begin{array}{l}\text { Gp1: EHM (HMDF) }(100 \%) \\
\text { Gp2: EPTF (BovF) }(0 \%)\end{array}$ & C1: Gp1 vs. Gp2 & $\begin{array}{l}\text { SS: Initiation of enteral } \\
\text { nutrition } \\
\text { SE: Earliest of } 91 \mathrm{~d} \text { of } \\
\text { age; DC; or } 50 \% \text { oral } \\
\text { feedings }\end{array}$ & $\begin{array}{l}\text { Growth (from regained } \\
\text { BW to SE): wt gain } \\
\text { (g/d), HC gain } \\
\text { (cm/wk), Length gain } \\
(\mathrm{cm} / \mathrm{wk})\end{array}$ & $\begin{array}{l}\text { Moderate } \\
\text { (sequence } \\
\text { generation and } \\
\text { allocation } \\
\text { concealment } \\
\text { unclear) }\end{array}$ \\
\hline $\begin{array}{l}\text { O'Connor } 2016 \text { [32] } \\
\text { Canada }\end{array}$ & $\mathrm{RCT}$ & $\begin{array}{l}363 \text { infants } \\
\text { Gp1: } 995 \pm 273 ; 27.5 \pm 2.4 ; 181 \\
\text { Gp2: } 996 \pm 272 ; 27.8 \pm 2.7 ; 182\end{array}$ & $\begin{array}{l}\text { Gp1: EHM (MOM + DHM) } \\
(100 \% \text {; MOM 58.4\% (13.6, } \\
\text { 96.0)) } \\
\text { Gp2: Mixed (MOM + PTF) } \\
\text { (MOM 63.3\% }(9.6,97.2))\end{array}$ & C3: Gp1 vs. Gp2 & $\begin{array}{l}\text { SS: Start enteral feeds } \\
\text { SE: } 90 \text { d or DC }\end{array}$ & $\begin{array}{l}\text { Growth (during } \\
\text { intervention): change in } \\
\text { wt, HC and length } \\
\text { z-score }\end{array}$ & Low \\
\hline $\begin{array}{l}\text { Schanler } 2005[33] \\
\text { USA }\end{array}$ & $\begin{array}{l}\text { RCT (randomised } \\
\text { arms) } \\
\text { Cohort } \\
\text { (non-randomised } \\
\text { arm) }\end{array}$ & $\begin{array}{l}243 \text { infants } \\
\text { Gp1: } 947 \pm 233 ; 27 \pm 2 ; 81 \\
\text { Gp2: } 957 \pm 267 ; 27 \pm 2 ; 92 \\
\text { Gp3: } 999 \pm 259 ; 27 \pm 2 ; 70\end{array}$ & $\begin{array}{l}\text { Gp1: EHM (MOM + DHM) } \\
(100 \%) \\
\text { Gp2: Mixed (MOM + PTF) } \\
\text { (NR) } \\
\text { Gp3: EHM (MOM) (100\%) } \\
\text { (reference, not randomised) }\end{array}$ & $\begin{array}{l}\text { C3 (RCT): Gp1 vs. Gp2 } \\
\text { C3 (non-RCT): Gp 1+ Gp3 vs. } \\
\text { Gp2 }\end{array}$ & $\begin{array}{l}\text { SS: } 4 \mathrm{~d} \text { after birth } \\
\text { SE: } 90 \mathrm{~d} \text { of age or DC }\end{array}$ & $\begin{array}{l}\text { Growth (during study): } \\
\text { wt gain }(\mathrm{g} / \mathrm{kg} / \mathrm{d}), \mathrm{HC} \\
\text { gain }(\mathrm{cm} / \mathrm{wk}), \text { Length } \\
\text { gain }(\mathrm{cm} / \mathrm{wk})\end{array}$ & $\begin{array}{l}\text { Low (sequence } \\
\text { generation } \\
\text { unclear) }\end{array}$ \\
\hline $\begin{array}{l}\text { Sullivan } 2010 \text { [34] } \\
\text { USA and Austria }\end{array}$ & $\mathrm{RCT}$ & $\begin{array}{l}207 \text { infants } \\
\text { Gp1: } 945 \pm 202 ; 27.2 \pm 2.2 ; 67 \\
\text { Gp2: } 909 \pm 193 ; 27.1 \pm 2.3 ; 71 \\
\text { Gp3: } 922 \pm 197 ; 27.3 \pm 2.0 ; 69\end{array}$ & $\begin{array}{l}\text { Gp1: EHM (MOM + DHM } \\
\text { + HMDF) (100\%; MOM } \\
73 \%(16,82))(\text { fortified at } \\
100 \mathrm{~mL} / \mathrm{kg} / \mathrm{d}) \\
\text { Gp2: } \mathrm{EHM}(\mathrm{MOM}+\mathrm{DHM} \\
\text { + HMDF) }(100 \% \text { MOM } \\
70 \%(18,80))(\text { fortified at } 40 \\
\mathrm{mL} / \mathrm{kg} / \mathrm{d}) \\
\text { Gp3: Mixed (MOM + PTF + } \\
\text { BovF) (NR; MOM } 82 \%(38, \\
100))(\text { fortified at } \\
100 \mathrm{mmL} / \mathrm{kg} / \mathrm{d})\end{array}$ & C $3:$ Gp1 12 vs. Gp 3 & $\begin{array}{l}\text { SS: Start of enteral } \\
\text { nutrition } \\
\text { SE: Earliest of } 91 \mathrm{~d} \text { of } \\
\text { age, DC, } 50 \% \text { oral } \\
\text { feedings }\end{array}$ & $\begin{array}{l}\text { Growth (from SS to SE): } \\
\text { mean wt gain } \\
\text { (g/kg/day), HC and } \\
\text { length gain (cm/wk); } \\
\mathrm{HC} \text { and length gain } \\
\text { reported as median, } \\
\text { IQR, and converted to } \\
\text { mean, SD. }\end{array}$ & $\begin{array}{l}\text { High (no blinding } \\
\text { of caregivers who } \\
\text { likely measured } \\
\text { growth) }\end{array}$ \\
\hline
\end{tabular}


Table 1. Cont.

\begin{tabular}{|c|c|c|c|c|c|c|c|}
\hline $\begin{array}{l}\text { Study } \\
\text { Country }\end{array}$ & Design & $\begin{array}{l}\text { Participants } \\
\text { BW, g; GA, wk; } n\end{array}$ & $\begin{array}{l}\text { Intervention } \\
\text { (Proportion of HM \% } \\
\text { Unless Stated Elsewhere) }\end{array}$ & $\begin{array}{l}\text { Comparisons for This } \\
\text { Review } \\
\text { C1: EHM vs. EPTF } \\
\text { C2: Any HM vs. EPTF } \\
\text { C3: High- vs. Low-Dose HM }\end{array}$ & Study Duration & Outcomes & Risk of Bias \\
\hline \multicolumn{8}{|l|}{ Observational Studies } \\
\hline $\begin{array}{l}\text { Assad } 2016[39] \\
\text { USA }\end{array}$ & $\begin{array}{l}\text { Interrupted time } \\
\text { series }\end{array}$ & $\begin{array}{l}293 \text { infants } \\
\text { BW range: } 490 \text { to } 1700 \\
\text { GA Gp1: } 27.7 \pm 2.7 ; 87 \\
\text { Gp2: } 28.3 \pm 2.8 ; 127 \\
\text { Gp3: } 27.6 \pm 2.8 ; 49 \\
\text { Gp4: } 29.8 \pm 2.5 ; 30\end{array}$ & $\begin{array}{l}\text { Gp1: EHM (MOM + DHM } \\
\text { + HMDF) (100\%) } \\
\text { Gp2: EHM (MOM + BovF) } \\
\text { (100\%) } \\
\text { Gp3: Mixed (MOM + BovF } \\
\text { + PTF) (NR) } \\
\text { Gp4: EPTF }(0 \%)\end{array}$ & $\begin{array}{l}\text { C1: Gp1 + Gp2 vs. Gp4 } \\
\text { C2: Gp1 + Gp2 + Gp3 vs. Gp4 } \\
\text { C3: Gp1 + Gp2 vs. G3 }\end{array}$ & NR & $\begin{array}{l}\text { Growth (from birth to } \\
\text { DC): wt gain }(\mathrm{g} / \mathrm{kg} / \mathrm{d})\end{array}$ & Low \\
\hline $\begin{array}{l}\text { Brownell } 2018 \text { [43] } \\
\text { USA }\end{array}$ & Cohort & $\begin{array}{l}314 \text { infants } \\
\text { Whole cohort: } 1233 \pm 373 \text {; } \\
29.5 \pm 2.9 ; 314\end{array}$ & $\begin{array}{l}10 \% \text { incremental exposure } \\
\text { to MOM, DHM, and PTF }\end{array}$ & C3: synthesised narratively & $\begin{array}{l}\text { SS: enteral feedings start } \\
\text { SE: } 36 \text { wk PMA or DC }\end{array}$ & $\begin{array}{l}\text { Growth (from birth to } \\
36 \text { wk PMA): wt gain } \\
\text { (g/kg/day), HC gain } \\
\text { (cm/wk), length gain } \\
\text { (cm/wk), change in wt, } \\
\text { length, and HC z-scores }\end{array}$ & Low \\
\hline $\begin{array}{l}\text { Canizo Vazquez } 2019 \\
\text { [44] Spain }\end{array}$ & Cohort & $\begin{array}{l}227 \text { infants } \\
\text { Gp 1: } 1283 \pm 393 ; 29.5 \pm 2.3 \\
99 \\
\text { Gp 2: } 1197 \pm 370 ; 29.1 \pm 2.3 \\
128\end{array}$ & $\begin{array}{l}\text { Gp1: MOM, PTF } \\
\text { Gp2: MOM, DHM }\end{array}$ & C3: Gp2 vs. Gp1 & Hospital stay & $\begin{array}{l}\text { Growth (from birth to } \\
\text { discharge): change in } \\
\text { wt z-score; length, and } \\
\text { HC z-score at SE }\end{array}$ & $\begin{array}{l}\text { Moderate } \\
\text { (Proportion of } \\
\text { HM not reported) }\end{array}$ \\
\hline $\begin{array}{l}\text { Carlson } 1998 \text { [45] } \\
\text { USA }\end{array}$ & Cohort & $\begin{array}{l}51 \text { infants } \\
\text { Whole cohort: } 899 \pm 205 ; 27.1 \\
\pm 1.9 ; 51\end{array}$ & $\begin{array}{l}\text { Gp1: EHM (MOM) (100\%) } \\
\text { Gp2: Mixed (MOM + PTF) } \\
\text { (NR) } \\
\text { Gp3: EPTF }(0 \%)\end{array}$ & $\begin{array}{l}\text { C1, C2, C3: synthesised } \\
\text { narratively }\end{array}$ & NR & $\begin{array}{l}\text { Growth (from birth to } \\
\text { DC): wt gain } \\
\text { (g/kg/day), measured } \\
\text { in time periods: } 0-14 \mathrm{~d} \text {, } \\
15-35 \mathrm{~d}, 36-56 \mathrm{~d}, 57 \mathrm{~d} \text { to } \\
\text { TCA }\end{array}$ & Low \\
\hline $\begin{array}{l}\text { Castellano Yanez } \\
2019 \text { [46] Spain }\end{array}$ & Cohort & $\begin{array}{l}130 \text { infants } \\
\text { Gp 1: } 1430 \pm 262 ; 31.2(30.1, \\
31.7) ; 52 \\
\text { Gp 2: } 1343 \pm 233 ; 31.5 \text { (30.0, } \\
\text { 32.7); } 78\end{array}$ & $\begin{array}{l}\text { Gp1: MOM, PTF } \\
\text { Gp2: MOM, DHM }\end{array}$ & C3: synthesised narratively & Hospital stay & $\begin{array}{l}\text { Growth (from birth to } \\
\text { DC): change in wt, } \\
\text { length, and HC } \\
\text { z-scores-difference } \\
\text { between groups }\end{array}$ & $\begin{array}{l}\text { Moderate (some } \\
\text { differences } \\
\text { between Gps) }\end{array}$ \\
\hline
\end{tabular}


Table 1. Cont.

\begin{tabular}{|c|c|c|c|c|c|c|c|}
\hline $\begin{array}{l}\text { Study } \\
\text { Country }\end{array}$ & Design & $\begin{array}{l}\text { Participants } \\
\text { BW, g; GA, wk; } n\end{array}$ & $\begin{array}{l}\text { Intervention } \\
\text { (Proportion of HM \% } \\
\text { Unless Stated Elsewhere) }\end{array}$ & $\begin{array}{l}\text { Comparisons for This } \\
\text { Review } \\
\text { C1: EHM vs. EPTF } \\
\text { C2: Any HM vs. EPTF } \\
\text { C3: High- vs. Low-Dose HM }\end{array}$ & Study Duration & Outcomes & Risk of Bias \\
\hline $\begin{array}{l}\text { Chowning } 2016 \text { [47] } \\
\text { USA }\end{array}$ & Cohort & $\begin{array}{l}550 \text { infants } \\
\text { Gp1: } 1030 \pm 290 ; 28.1 \pm 2.7 ; \\
260 \\
\text { Gp2: } 1080 \pm 280 ; 28.6 \pm 2.5 ; \\
\text { 290 } \\
\text { Gp3: } 1150 \pm 290 ; 29.3 \pm 2.8 ; 76 \\
\text { Gp4: } 1160 \pm 240 ; 29.1 \pm 2.4 ; 71\end{array}$ & $\begin{array}{l}\text { Gp1: }<50 \% \text { d received HM } \\
\text { Gp2: } \geq 50 \% \text { d received HM } \\
\text { Separate analysis done for } \\
\text { extremes of intake } \\
\text { Gp3: } 0 \% \text { d received HM } \\
\text { Gp4: } \geq 90 \% \text { d received HM }\end{array}$ & $\begin{array}{l}\text { C2: Gp4 vs. Gp3 } \\
\text { C3: Gp2 vs. Gp1 }\end{array}$ & Hospital stay & $\begin{array}{l}\text { Growth: wt gain (from } \\
\text { regained BW to DC, } \\
\text { g/kg/d), HC gain (from } \\
\text { birth to DC, cm } / \text { wk), } \\
\text { Change in wt and HC } \\
\text { z-score (from birth to } \\
\text { DC) }\end{array}$ & Low \\
\hline $\begin{array}{l}\text { Colacci } 2017[40] \\
\text { USA }\end{array}$ & $\begin{array}{l}\text { Interrupted time } \\
\text { series }\end{array}$ & $\begin{array}{l}85 \text { infants } \\
\text { Gp1: } 783 \pm 143 ; 26.0 \pm 1.9 ; 39 \\
\text { Gp2: } 770 \pm 137 ; 26.0 \pm 1.9 ; 46\end{array}$ & $\begin{array}{l}\text { Gp1: EHM (MOM + DHM } \\
+ \text { HMDF) (100\%) } \\
\text { Gp2: Mixed (MOM + PTF + } \\
\text { BovF) ( } 83 \% \text { of feeding as } \\
\text { formula) }\end{array}$ & C3: Gp1 vs. Gp2 & $\begin{array}{l}\text { SS: birth } \\
\text { SE: at least for } 4 \mathrm{wk} \text { and } \\
\text { wt } \geq 1500 \mathrm{~g} \text { or } 34 \mathrm{wk} \\
\text { PMA (whichever occur } \\
\text { first) }\end{array}$ & $\begin{array}{l}\text { Growth (from birth to } \\
\text { DC): wt gain }(\mathrm{g} / \mathrm{kg} / \mathrm{d})\end{array}$ & Low \\
\hline $\begin{array}{l}\text { Colaizy } 2012[7] \\
\text { USA }\end{array}$ & Cohort & $\begin{array}{l}171 \text { infants } \\
\text { Gp1: } 1083(778,1184) ; 28.4 \\
(25.4,29.6) ; 17 \\
\text { Gp2: } 861(736,1091) ; \\
\text { 26.89(25.4, 29.0); 30 } \\
\text { Gp3: } 848(717,1011) ; 26.6(25.7, \\
\text { 28.5); } 36 \\
\text { Gp4: } 880(719,1052) ; 27(25.6, \\
\text { 28.8); } 88\end{array}$ & $\begin{array}{l}\text { Gp1: }<25 \% \text { HM }(\mathrm{MOM}+ \\
\text { DHM + PTF) } \\
\text { Gp2: } 25-50 \% \text { HM }(\mathrm{MOM}+ \\
\text { DHM + PTF) } \\
\text { Gp3: 51-75\% HM (MOM + } \\
\text { DHM + PTF) } \\
\text { Gp4: > 75\% HM (MOM + } \\
\text { DHM + PTF) }\end{array}$ & C3: Gp1 + Gp2 vs. Gp3 + Gp4 & Hospital stay & $\begin{array}{l}\text { Growth (from birth to } \\
\text { DC): change in wt } \\
\text { z-score (reported as } \\
\text { median, IQR and } \\
\text { converted to mean, SD) }\end{array}$ & Low \\
\hline $\begin{array}{l}\text { Costa-Orvay } 2011 \\
{[15]} \\
\text { Spain }\end{array}$ & $\begin{array}{l}\text { RCT with } \\
\text { non-randomised } \\
\text { reference group }\end{array}$ & $\begin{array}{l}38 \text { infants } \\
\text { Gp1: } 1138 \pm 173 ; 29.0 \pm 1.7 ; 6 \\
\text { Gp2: } 1196 \pm 243 ; 29.6 \pm 1.6 ; 8 \\
\text { Gp3: } 1220 \pm 221 ; 30.2 \pm 1.4 ; 12 \\
\text { Gp4: } 1313 \pm 336 ; 29.8 \pm 1.7 ; 12\end{array}$ & $\begin{array}{l}\text { Gp1: EHM (MOM + BovF) } \\
\text { (100\%), } \\
\text { (reference, not randomised) } \\
\text { Gp2: EPTF }(0 \%) \\
\text { Gp3: EPTF (high energy } \\
\text { and protein formula) }(0 \%) \\
\text { Gp4: EPTF (high energy } \\
\text { and protein formula) }(0 \%)\end{array}$ & C1: Gp1 vs. Gp2 & $\begin{array}{l}\text { Intervention: } 4 \mathrm{wks} \\
\text { from regain BW }\end{array}$ & $\begin{array}{l}\text { Body composition (BIA) } \\
\text { at SE: FM (g), FFM (g) }\end{array}$ & Low \\
\hline
\end{tabular}


Table 1. Cont.

\begin{tabular}{|c|c|c|c|c|c|c|c|}
\hline $\begin{array}{l}\text { Study } \\
\text { Country }\end{array}$ & Design & $\begin{array}{l}\text { Participants } \\
\text { BW, g; GA, wk; } n\end{array}$ & $\begin{array}{l}\text { Intervention } \\
\text { (Proportion of HM \% } \\
\text { Unless Stated Elsewhere) }\end{array}$ & $\begin{array}{l}\text { Comparisons for This } \\
\text { Review } \\
\text { C1: EHM vs. EPTF } \\
\text { C2: Any HM vs. EPTF } \\
\text { C3: High- vs. Low-Dose HM }\end{array}$ & Study Duration & Outcomes & Risk of Bias \\
\hline $\begin{array}{l}\text { Fewtrell } 2002 \text { [19] } \\
\text { United Kingdom }\end{array}$ & $\begin{array}{l}\text { RCT with } \\
\text { non-randomised } \\
\text { reference group }\end{array}$ & $\begin{array}{l}283 \text { infants } \\
\text { Gp1: } 1353 \pm 274 ; 30.3 \pm 2.4 ; \\
100 \\
\text { Gp2: } 1336 \pm 284 ; 30.4 \pm 2.3 ; 95 \\
\text { Gp3: } 1395 \pm 262 ; 30.3 \pm 2.0 ; 88\end{array}$ & $\begin{array}{l}\text { Gp1: Control PTF }(0 \%) \\
\text { Gp2: } \\
\text { LCPUFA-supplemented } \\
\text { formula }(0 \%) \\
\text { Gp3: MOM (NR) } \\
\text { (reference, not randomised) }\end{array}$ & C2: Gp3 vs. Gp1 & $\begin{array}{l}\text { SS: } 10 \mathrm{~d} \text { of age } \\
\text { SE: } 18 \mathrm{~m} \mathrm{CA}\end{array}$ & $\begin{array}{l}\text { Growth (from birth to } \\
\text { DC): wt gain }(\mathrm{g} / \mathrm{kg} / \mathrm{d}) \text {, } \\
\text { HC gain }(\mathrm{cm} / \mathrm{wk})\end{array}$ & Low \\
\hline $\begin{array}{l}\text { Ginovart } 2017 \text { [8] } \\
\text { Spain }\end{array}$ & $\begin{array}{l}\text { Interrupted time } \\
\text { series }\end{array}$ & $\begin{array}{l}182 \text { infants } \\
\text { Gp1: } 1108 \pm 273 ; 29^{+4} \pm 2^{+6} \\
72 \\
\text { Gp2: } 1078 \pm 289 ; 29^{+1} \pm 2^{+6} \\
114\end{array}$ & $\begin{array}{l}\text { Gp1: Any PTF (PTF + } \\
\text { MOM) (NR) } \\
\text { Gp2: EHM (MOM + DHM } \\
+ \text { BovF) }(100 \%)\end{array}$ & C3: Gp2 vs. Gp1 & Hospital stay & $\begin{array}{l}\text { Growth (from birth to } \\
\text { DC): change in wt and } \\
\text { HC z-score }\end{array}$ & Low \\
\hline $\begin{array}{l}\text { Hair } 2016 \text { [48] } \\
\text { USA }\end{array}$ & Cohort & $\begin{array}{l}1587 \text { infants } \\
\text { Gp1: } 823 \pm 205 ; 26.4 \pm 2.3 ; 768 \\
\text { Gp2: } 844 \pm 210 ; 26.5 \pm 2.5 ; 819\end{array}$ & $\begin{array}{l}\text { Gp1: MOM + BovF + PTF } \\
\text { (NR) } \\
\text { Gp2: EHM (MOM+ DHM + } \\
\text { HMDF) }(100 \%)\end{array}$ & C3: Gp2 vs. Gp1 & $\begin{array}{l}\text { SS: NR } \\
\text { SE: Varied between } \\
\text { sites: } 34 \text { wk PMA, } 60 \mathrm{~d} \\
\text { of age, at } 1500 \mathrm{~g} \text { or } 34 \\
\text { wk PMA, } 32 \text { wk PMA }\end{array}$ & $\begin{array}{l}\text { Growth (time frame for } \\
\text { measures NR): wt gain } \\
\text { (g/d), HC gain } \\
(\mathrm{cm} / \mathrm{wk})\end{array}$ & $\begin{array}{l}\text { Moderate (some } \\
\text { differences } \\
\text { between Gps) }\end{array}$ \\
\hline $\begin{array}{l}\text { Huston } 2014 \text { [29] } \\
\text { USA }\end{array}$ & Cohort & $\begin{array}{l}361 \text { infants } \\
\text { Gp1: } 1177 \pm 222 ; 29.1 \pm 1.8 ; 93 \\
\text { Gp2: } 1104 \pm 262 ; 28.1 \pm 2.2 ; \\
224 \\
\text { Gp3: } 919 \pm 269 ; 26.7 \pm 2.4 ; 44\end{array}$ & $\begin{array}{l}\text { Gp1: EPTF or MOM + } \\
\text { BovF + PTF for }>48 \mathrm{~h}(\mathrm{NR}) \\
\text { Gp2: MOM + DHM + BovF } \\
(100 \%) \\
\text { Gp3: EHM (MOM + DHM } \\
+ \text { HMDF }(100 \%)\end{array}$ & C3: Gp2 + Gp3 vs. Gp1 & NR & $\begin{array}{l}\text { Growth (during } \\
\text { hospital stay): wt gain } \\
(\mathrm{g} / \mathrm{kg} / \mathrm{d}), \text { HC gain } \\
(\mathrm{cm} / \mathrm{wk}) \text {, length gain } \\
(\mathrm{cm} / \mathrm{wk})\end{array}$ & $\begin{array}{l}\text { Moderate (some } \\
\text { differences } \\
\text { between Gps) }\end{array}$ \\
\hline
\end{tabular}


Table 1. Cont.

\begin{tabular}{|c|c|c|c|c|c|c|c|}
\hline $\begin{array}{l}\text { Study } \\
\text { Country }\end{array}$ & Design & $\begin{array}{l}\text { Participants } \\
\text { BW, g; GA, wk; } n\end{array}$ & $\begin{array}{l}\text { Intervention } \\
\text { (Proportion of HM \% } \\
\text { Unless Stated Elsewhere) }\end{array}$ & $\begin{array}{l}\text { Comparisons for This } \\
\text { Review } \\
\text { C1: EHM vs. EPTF } \\
\text { C2: Any HM vs. EPTF } \\
\text { C3: High- vs. Low-Dose HM }\end{array}$ & Study Duration & Outcomes & Risk of Bias \\
\hline $\begin{array}{l}\text { Huston } 2018[28] \\
\text { USA } \\
\text { Some overlap of } \\
\text { infants between this } \\
\text { report and Huston } \\
2014\end{array}$ & $\begin{array}{l}\text { Interrupted time } \\
\text { series }\end{array}$ & $\begin{array}{l}379 \text { infants } \\
\text { Gp1: } 1025 \pm 164 ; 28.4 \pm 1.9 ; 54 \\
\text { Gp2: } 944 \pm 199 ; 27.4 \pm 2.0 ; 87 \\
\text { Gp3: } 959 \pm 174 ; 27.1 \pm 2.0 ; 111 \\
\text { Gp4: } 855 \pm 209 ; 26.2 \pm 2.2 ; 33 \\
\text { Gp5: } 904 \pm 200 ; 26.6 \pm 2.4 ; 94\end{array}$ & $\begin{array}{l}\text { Gp1: PTF (MOM + PTF + } \\
\text { BovF) (NR) } \\
\text { Gp2: HMBF1 (MOM + } \\
\text { DHM + BovF) (before } \\
\text { implementation of the } \\
\text { feeding protocol) (100\%) } \\
\text { Gp3: HMBF2 (MOM + } \\
\text { DHM + BovF) (after } \\
\text { implementation of the } \\
\text { protocol) } \\
\text { Gp4: EHM1 (MOM + } \\
\text { HMDF) (before } \\
\text { implementation of the } \\
\text { feeding protocol) (100\%) } \\
\text { Gp5: EHM2 (MOM + } \\
\text { HMDF) (after } \\
\text { implementation of the } \\
\text { feeding protocol) }\end{array}$ & C3: Gp2 + Gp4 vs. Gp1 & Hospital stay & $\begin{array}{l}\text { Growth (during } \\
\text { hospital stay): change in } \\
\text { wt, HC and length } \\
\text { z-score }\end{array}$ & $\begin{array}{l}\text { Moderate (some } \\
\text { differences } \\
\text { between Gps) }\end{array}$ \\
\hline $\begin{array}{l}\text { Jacobi-Polishook } \\
2016[37] \\
\text { Australia }\end{array}$ & $\begin{array}{l}\text { Secondary } \\
\text { analysis of RCT }\end{array}$ & $\begin{array}{l}611 \text { infants } \\
\text { median (range) } \\
\text { Gp1: } 1580 \text { (720, 2280); } 31.0 \\
(25.0,32.0) ; 46 \\
\text { Gp2: } 1360(530,2620) ; 30.0 \\
(24.0,32.0) ; 141 \\
\text { Gp3: } 1390(420,2400) ; 30.0 \\
(23.0,33.0) ; 141 \\
\text { Gp4: } 1290(500,2090) ; 29.0 \\
(23.0,33.0) ; 142 \\
\text { Gp5: } 1240(320,2480) ; 30.0 \\
(24.0,33.0) ; 141\end{array}$ & $\begin{array}{l}\text { Gp1: EPTF }(0 \%) \\
\text { Gp2: Q1 (HM median } \\
\text { (range) 49 }(0.1,85) \\
\text { mL/kg/d) } \\
\text { Gp3: Q2 (HM } 103(85,114) \\
\text { ml/kg/d) } \\
\text { Gp4: Q3 (HM } 124(114,134) \\
\text { ml/kg/d) } \\
\text { Gp5: Q4 (HM } 149(134,180) \\
\text { ml/kg/d) }\end{array}$ & $\begin{array}{l}\text { C2: Gp2 + Gp3 + Gp4 + Gp5 } \\
\text { vs. G1 } \\
\text { C3: Gp4 + Gp5 vs. Gp2 + Gp3 } \\
\text { BMI gain synthesised } \\
\text { narratively }\end{array}$ & Hospital stay & $\begin{array}{l}\text { Growth (from birth to } \\
\text { DC): length gain } \\
\text { (cm/wk), BMI gain }\end{array}$ & $\begin{array}{l}\text { Moderate (some } \\
\text { differences } \\
\text { between Gps) }\end{array}$ \\
\hline $\begin{array}{l}\text { Kaempf } 1998 \text { [36] } \\
\text { Germany }\end{array}$ & $\begin{array}{l}\text { Non-randomised } \\
\text { trial }\end{array}$ & $\begin{array}{l}19 \text { infants } \\
\text { Gp1: } 1220 \pm 310 ; 29 \pm 1.1 ; 11 \\
\text { Gp2: } 1220 \pm 270 ; 30 \pm 1.6 ; 8\end{array}$ & $\begin{array}{l}\text { Gp1: PTF ( }>80 \% \text { PTF) } \\
\text { Gp2: HM (fortified MOM) } \\
\text { (>80\% fortified MOM) }\end{array}$ & $\begin{array}{l}\text { C3: Gp1 vs. Gp2 for wt gain } \\
\text { (g/d); } \\
\text { lower leg length synthesised } \\
\text { narratively }\end{array}$ & $\begin{array}{l}\text { SS: Gp1; age } 8 \pm 5 \mathrm{~d} \text {, } \\
\text { Gp2; age } 10 \pm 5 \mathrm{~d} \\
\text { Study duration: Gp1; } 51 \\
\pm 12 \mathrm{~d}, \mathrm{Gp} 2 ; 48 \pm 18 \mathrm{~d}\end{array}$ & $\begin{array}{l}\text { Growth (time frame for } \\
\text { measures NR): wt gain } \\
(\mathrm{g} / \mathrm{d}) \text {, lower leg length } \\
(\mathrm{mm} / \mathrm{d})\end{array}$ & $\begin{array}{l}\text { Moderate (some } \\
\text { differences } \\
\text { between Gps) }\end{array}$ \\
\hline
\end{tabular}


Table 1. Cont.

\begin{tabular}{|c|c|c|c|c|c|c|c|}
\hline $\begin{array}{l}\text { Study } \\
\text { Country }\end{array}$ & Design & $\begin{array}{l}\text { Participants } \\
\text { BW, g; GA, wk; } n\end{array}$ & $\begin{array}{l}\text { Intervention } \\
\text { (Proportion of HM \% } \\
\text { Unless Stated Elsewhere) }\end{array}$ & $\begin{array}{l}\text { Comparisons for This } \\
\text { Review } \\
\text { C1: EHM vs. EPTF } \\
\text { C2: Any HM vs. EPTF } \\
\text { C3: High- vs. Low-Dose HM }\end{array}$ & Study Duration & Outcomes & Risk of Bias \\
\hline $\begin{array}{l}\text { Lee } 2020 \text { [49] } \\
\text { Singapore, Malaysia }\end{array}$ & Cohort & $\begin{array}{l}236 \text { infants } \\
\text { Gp 1: } 855 \pm 123 ; 27.1 \pm 1.9 ; 40 \\
\text { Gp 2: } 849 \pm 120 ; 27.6 \pm 2.4 ; 34\end{array}$ & $\begin{array}{l}\text { Gp1 (Singapore): MOM } \\
\text { 97\% } \\
\text { Gp2 (Malaysia): MOM } \\
\text { 26\%, Mix MOM/PTF } 62 \% \text {, } \\
\text { EPTF } 12 \%\end{array}$ & C3: Gp1 vs. Gp2 & Birth to 36 wk PMA & $\begin{array}{l}\text { Growth (birth to } 36 \mathrm{wk} \\
\text { PMA): change in wt } \\
\text { z-score }\end{array}$ & Low \\
\hline $\begin{array}{l}\text { Li } 2019[38] \\
\text { United Kingdom }\end{array}$ & $\begin{array}{l}\text { Secondary } \\
\text { analysis of RCT }\end{array}$ & $\begin{array}{l}133 \text { infants } \\
\text { Gp1: } 997(780,1178) ; 28.1(26.5 \text {, } \\
\text { 29.5); } 56 \\
\text { Gp2: } 1140(885,1398) ; 28.6 \\
(26.8,30.1) ; 39 \\
\text { Gp3: } 1132(905,1334) ; 28.2 \\
(26.9,30.0) ; 38\end{array}$ & $\begin{array}{l}\text { (RCT: four PN intervention } \\
\text { groups) } \\
\text { Gp1: EHM; MOM (100\%) } \\
\text { Gp2: Predominantly HM; } \\
\text { MOM + DHM + PTF (NR) } \\
\text { Gp3: Predominantly PTF; } \\
\text { MOM + DHM + PTF (NR) }\end{array}$ & $\begin{array}{l}\text { C3: \% FFM Gp1 + Gp2 vs. Gp } \\
\text { 3; remainder of outcomes } \\
\text { synthesised narratively }\end{array}$ & $\begin{array}{l}\text { Nutritional intake: from } \\
\text { birth until } 34 \text { wks PMA }\end{array}$ & $\begin{array}{l}\text { Growth (birth to TCA): } \\
\text { wt, length, and HC } \\
\text { change in z-scores } \\
\text { Body composition } \\
\text { (MRI) at TCA: FM (g } \\
\text { and \%), FFM (g), FFM \% } \\
\text { (reported as median } \\
\text { IQR and converted to } \\
\text { mean, SD) }\end{array}$ & Low \\
\hline $\begin{array}{l}\text { Lok } 2017 \text { [22] } \\
\text { Hong Kong }\end{array}$ & Cohort & $\begin{array}{l}\text { 175 VLBW infants } \\
\text { Gp1: } 1269.3 \pm 180.6 ; \mathrm{NR} ; 31 \\
\text { Gp2: } 1139.2 \pm 205.9 ; \mathrm{NR} ; 144 \\
\text { Gp3: } 1213 \pm 204.3 ; \mathrm{NR} ; 55 \\
\text { Gp4: } 1202 \pm 189.5 ; \mathrm{NR} ; 25 \\
\text { Gp5: } 1135 \pm 221.5 ; \mathrm{NR} 47 \\
\text { Gp6: } 1106 \pm 191.4 ; \mathrm{NR} ; 47\end{array}$ & $\begin{array}{l}\text { Gp1: EPTF }(0 \%) \\
\text { Gp2: Any HM (NR) } \\
\text { Group by proportion of } \\
\text { breast milk intake } \\
\text { Gp3: }<25 \% \mathrm{HM} \\
\text { Gp4: } 25-50 \% \mathrm{HM} \\
\text { Gp5: } 50-75 \% \mathrm{HM} \\
\text { Gp6: }>75 \% \mathrm{HM}\end{array}$ & $\begin{array}{l}\text { C2: Gp2 vs. Gp1 } \\
\text { C3: Gp5 + Gp6 vs. Gp3 + Gp4 }\end{array}$ & $\begin{array}{l}\text { The first } 30 \mathrm{~d} \text { of } \\
\text { hospitalization }\end{array}$ & $\begin{array}{l}\text { Growth (from birth to } \\
\text { DC): change in wt and } \\
\text { HC z-score }\end{array}$ & Low \\
\hline $\begin{array}{l}\text { Maas } 2013[51] \\
\text { Germany }\end{array}$ & Cohort & $\begin{array}{l}206 \text { infants } \\
\text { Gp1: } 846 \text { (705, 1160); } 28.6(25.5, \\
\text { 30.5); } 37 \\
\text { Gp2: } 925(665,1175) ; 27.6(25.5 \text {, } \\
\text { 29.7); } 122\end{array}$ & $\begin{array}{l}\text { Gp1: }<25 \% \text { HM } \\
\text { Gp2: }>75 \% \text { HM }\end{array}$ & C3: Gp 2 vs. Gp1 & Hospital stay & $\begin{array}{l}\text { Growth (from birth to } \\
\text { day 28): wt and HC } \\
\text { changes in z-score } \\
\text { (reported as median, } \\
\text { IQR and converted to } \\
\text { mean, SD) }\end{array}$ & $\begin{array}{l}\text { Moderate (some } \\
\text { loss to FU) }\end{array}$ \\
\hline
\end{tabular}


Table 1. Cont.

\begin{tabular}{|c|c|c|c|c|c|c|c|}
\hline $\begin{array}{l}\text { Study } \\
\text { Country }\end{array}$ & Design & $\begin{array}{l}\text { Participants } \\
\text { BW, g; GA, wk; } n\end{array}$ & $\begin{array}{l}\text { Intervention } \\
\text { (Proportion of HM \% } \\
\text { Unless Stated Elsewhere) }\end{array}$ & $\begin{array}{l}\text { Comparisons for This } \\
\text { Review } \\
\text { C1: EHM vs. EPTF } \\
\text { C2: Any HM vs. EPTF } \\
\text { C3: High- vs. Low-Dose HM }\end{array}$ & Study Duration & Outcomes & Risk of Bias \\
\hline $\begin{array}{l}\text { Madore } 2017 \text { [52] } \\
\text { USA }\end{array}$ & Cohort & $\begin{array}{l}81 \text { infants } \\
\text { Gp1: } 936.6 \pm 211.0 ; 27.0 \pm 1.5 ; \\
29 \\
\text { Gp2: } 913.8 \pm 222.6 ; 27.3 \pm 2.1 ; \\
25 \\
\text { Gp3: } 890.5 \pm 175.8 ; 27.1 \pm 1.9 ; \\
27\end{array}$ & $\begin{array}{l}\text { Gp1: EHM; MOM }(100 \%) \\
\text { Gp2: Predominantly PTF } \\
\text { (>50\% PTF) } \\
\text { Gp3: DHM (>50\% DHM) }\end{array}$ & C3: Gp1 + Gp3 vs. Gp2 & The first month of life & $\begin{array}{l}\text { Growth (from birth to } \\
\text { day } 30 \text { and } 60) \text { : wt gain } \\
\text { (g/kg/d), HC gain } \\
(\mathrm{cm} / \mathrm{wk}) \text {, length gain } \\
(\mathrm{cm} / \mathrm{wk})\end{array}$ & Low \\
\hline $\begin{array}{l}\text { Manea } 2016 \text { [53] } \\
\text { Romania }\end{array}$ & Cohort & $\begin{array}{l}34 \text { infants } \\
\text { Birth wt range; } 850-1000 \mathrm{~g} \\
\text { Birth GA; } 25-33 \text { weeks } \\
\text { Gp1: } n=16 \\
\text { Gp2: } n=18\end{array}$ & $\begin{array}{l}\text { Gp1: EPTF }(0 \%) \\
\text { Gp2: EHM; MOM + BovF } \\
\text { (after reached } 100 \\
\mathrm{~mL} / \mathrm{kg} / \text { day) }(100 \%)\end{array}$ & C1: synthesised narratively & Hospital stay & $\begin{array}{l}\text { Growth (from birth to } 5 \\
\text { wk of age): wt gain } \\
\text { (g/d) }\end{array}$ & $\begin{array}{l}\text { High (Gp } \\
\text { characteristics } \\
\text { and participant } \\
\text { flow not } \\
\text { described. SD } \\
\text { and } p \text { values NR) }\end{array}$ \\
\hline $\begin{array}{l}\text { Mol } 2019[54] \\
\text { Poland }\end{array}$ & Cohort & $\begin{array}{l}53 \text { infants } \\
\text { BW; mean } \pm \text { SD, GA; median } \\
\text { (IQR) } \\
\text { Gp1: } 1240 \pm 180 ; 29 \text { (28-31.8); } \\
23 \\
\text { Gp2: } 1210 \pm 161 ; 29 \text { (28-32); } \\
11 \\
\text { Gp3: } 3320 \pm 399 ; 39 \text { (37-40); } \\
19\end{array}$ & $\begin{array}{l}\text { Gp1: } \operatorname{EPTF}(0 \%) \\
\text { Gp2: Fortified HM (MOM } \\
+ \text { BovF) }(100 \%) \\
\text { Gp3: full-term infants }\end{array}$ & C1: Gp2 vs. Gp1 & Hospital stay & $\begin{array}{l}\text { Body composition (BIS) } \\
\text { at TCA: FM (g and \%), } \\
\text { FFM (g and \%) }\end{array}$ & $\begin{array}{l}\text { Moderate (milk } \\
\text { amount not } \\
\text { described) }\end{array}$ \\
\hline $\begin{array}{l}\text { Morlacchi } 2018 \text { [55] } \\
\text { Italy }\end{array}$ & Cohort & $\begin{array}{l}32 \text { infants } \\
\text { Gp1: } 1214.8 \pm 246 ; 29.2 \pm 1.6 \\
17 \\
\text { Gp2: } 1293.0 \pm 138 ; 30.3 \pm 1 ; 15\end{array}$ & $\begin{array}{l}\text { Gp1: Fortified HM (MOM } \\
+ \text { BovF) }(100 \%) \\
\text { Gp2: EPTF }(0 \%)\end{array}$ & $\begin{array}{l}\text { C1: Gp1 vs. Gp2 } \\
\text { Change in z-scores } \\
\text { synthesised narratively }\end{array}$ & $\begin{array}{l}\text { SS: at DC } \\
\text { SE: at TCA } \\
\text { Intervention start from } \\
\text { birth to DC }\end{array}$ & $\begin{array}{l}\text { Growth (from birth to } \\
\text { DC): wt, length, and HC } \\
\text { change in z-scores } \\
\text { Body composition (ADP } \\
\text { (PeaPod) at DC): FM (g } \\
\text { and \%), FFM (g and \%) }\end{array}$ & Low \\
\hline $\begin{array}{l}\text { Nicholl } 1999 \text { [35] } \\
\text { United Kingdom }\end{array}$ & $\begin{array}{l}\text { RCT with } \\
\text { non-randomised } \\
\text { arm }\end{array}$ & $\begin{array}{l}52 \text { infants } \\
\text { Gp1: } 1074 \pm 216 ; 29 \pm 2.1 ; 10 \\
\text { Gp2: } 1002 \pm 286 ; 28.1 \pm 2.4 ; 13 \\
\text { Gp3: } 1087 \pm 252 ; 28.7 \pm 2.5 ; 29\end{array}$ & $\begin{array}{l}\text { Gp1: EHM (MOM + DHM) } \\
(100 \%) \\
\text { Gp2: EHM (MOM + DHM } \\
\text { + BovF) }(100 \%) \\
\text { Gp3: EPTF }(0 \%) \text { (not } \\
\text { randomised) }\end{array}$ & $\begin{array}{l}\text { C1: Gp2 vs. Gp3 } \\
\text { Lower leg length synthesised } \\
\text { narratively }\end{array}$ & $\begin{array}{l}\text { SS: enteral feed } \geq 150 \\
\mathrm{~mL} / \mathrm{kg} / \text { day } \\
\text { SE: nasogastric feeds } \\
\text { ceased }\end{array}$ & $\begin{array}{l}\text { Growth (from SS to SE): } \\
\text { wt gain }(\mathrm{g} / \mathrm{kg} / \mathrm{d}) \text {, lower } \\
\text { leg length gain }\end{array}$ & Low \\
\hline
\end{tabular}


Table 1. Cont.

\begin{tabular}{|c|c|c|c|c|c|c|c|}
\hline $\begin{array}{l}\text { Study } \\
\text { Country }\end{array}$ & Design & $\begin{array}{l}\text { Participants } \\
\text { BW, g; GA, wk; } n\end{array}$ & $\begin{array}{l}\text { Intervention } \\
\text { (Proportion of HM \% } \\
\text { Unless Stated Elsewhere) }\end{array}$ & $\begin{array}{l}\text { Comparisons for This } \\
\text { Review } \\
\text { C1: EHM vs. EPTF } \\
\text { C2: Any HM vs. EPTF } \\
\text { C3: High- vs. Low-Dose HM }\end{array}$ & Study Duration & Outcomes & Risk of Bias \\
\hline $\begin{array}{l}\text { O'Connor } 2001[20] \\
\text { USA and United } \\
\text { Kingdom }\end{array}$ & $\begin{array}{l}\text { RCT with } \\
\text { non-randomised } \\
\text { reference group }\end{array}$ & $\begin{array}{l}470 \text { infants } \\
\text { Gp1: } 1287 \pm 272 ; 29.6 \pm 1.9 ; \\
142 \\
\text { Gp2: } 1305 \pm 293 ; 29.8 \pm 2.1 ; \\
138 \\
\text { Gp3: } 1309 \pm 286 ; 29.7 \pm 2.0 ; \\
\text { 140 } \\
\text { Gp4: } 1275 \pm 312 ; 29.7 \pm 2.1 ; 43\end{array}$ & $\begin{array}{l}\text { Gp1: HM + PTF (NR) } \\
\text { Gp2: HM + PTF + AA + } \\
\text { DHA from fish/fungal oil } \\
\text { Gp3: HM + PTF + AA + } \\
\text { DHA from egg-derived } \\
\text { triglyceride/ fish oil } \\
\text { G4: HM ( }>80 \% \text { at term } \\
\text { corrected age) (reference, } \\
\text { not randomised) }\end{array}$ & C3: Gp4 vs. Gp1 & $\begin{array}{l}\text { SS: first enteral feed } \\
\text { SE: } 12 \mathrm{~m} \mathrm{CA}\end{array}$ & $\begin{array}{l}\text { Growth (from SS to } \\
\text { TCA): wt gain }(\mathrm{g} / \mathrm{kg} / \mathrm{d}) \text {, } \\
\mathrm{HC} \text { gain }(\mathrm{cm} / \mathrm{wk}), \\
\text { length gain }(\mathrm{cm} / \mathrm{wk})\end{array}$ & Low \\
\hline $\begin{array}{l}\text { Petrova } 2020 \text { [56] } \\
\text { USA }\end{array}$ & Cohort & $\begin{array}{l}84 \text { infants } \\
\text { Gp 1: } 1027 \text { (95\% CI 924, 1321); } \\
27.7 \text { (95\% CI 26.8, 28.6); } 37 \\
\text { Gp 2: } 1285 \text { (95\% CI 1130, 1439); } \\
\text { 29.8 (95\% CI 28.6, 31.0); } 16 \\
\text { Gp 3: } 1272 \text { (95\% CI 1102, 1442); } \\
29.1(95 \% \text { CI 28.2, 30.3); } 31\end{array}$ & $\begin{array}{l}\text { Gp1: Predominantly HM } \\
\text { ( } \geq 97 \% \text { HM) } \\
\text { Gp2: Partial HM }(55-70 \% \\
\text { HM) } \\
\text { Gp3: Predominantly PTF } \\
\text { ( } \leq 9 \% \text { HM) }\end{array}$ & C3: Gp1 + Gp2 vs. Gp3 & $\begin{array}{l}\text { SS: full enteral feeding } \\
\text { had been achieved } \\
\text { SE: } 2 \text { wks post full } \\
\text { enteral feeds }\end{array}$ & $\begin{array}{l}\text { Growth (from SS to SE): } \\
\text { wt gain }(\mathrm{g} / \mathrm{kg} / \mathrm{d} \text { ) } \\
\text { reported as median, } \\
\text { IQR in figure format } \\
\text { only; HC gain (cm } / \mathrm{wk}) \\
\text { reported as mean, } 95 \% \\
\mathrm{CI} \text { and converted to } \\
\text { mean, SD }\end{array}$ & $\begin{array}{l}\text { Moderate (some } \\
\text { differences } \\
\text { between Gps) }\end{array}$ \\
\hline $\begin{array}{l}\text { Pieltain } 2001 \text { [57] } \\
\text { Belgium }\end{array}$ & Cohort & $\begin{array}{l}54 \text { infants } \\
\text { Gp1: } 1298 \pm 317 ; 31 \pm 2 ; 20 \\
\text { Gp2: } 1269 \pm 261 ; 30 \pm 2 ; 34\end{array}$ & $\begin{array}{l}\text { Gp1: Fortified HM; MOM + } \\
\text { DHM + BovF }(100 \%) \\
\text { Gp2: EPTF }(0 \%)\end{array}$ & C1: Gp1 vs. Gp2 & $\begin{array}{l}\text { SS: full enteral feeding } \\
\text { had been achieved } \\
\text { SE: at DC }\end{array}$ & $\begin{array}{l}\text { Growth (from SS to SE): } \\
\text { wt gain }(\mathrm{g} / \mathrm{kg} / \mathrm{d}), \mathrm{HC} \\
\text { gain }(\mathrm{cm} / \mathrm{wk}), \text { length } \\
\text { gain }(\mathrm{cm} / \mathrm{wk}) \\
\text { Body composition } \\
\text { (DEXA at SS and } \\
\text { around DC): FFM (g), } \\
\text { FM (g and \%) }\end{array}$ & Low \\
\hline $\begin{array}{l}\text { Piemontese, } 2018 \\
\text { [58] } \\
\text { Italy }\end{array}$ & Cohort & $\begin{array}{l}73 \text { infants } \\
\text { Gp1: } 1207 \pm 208 ; 30 \pm 2.4 ; 24 \\
\text { Gp2: } 1269 \pm 193 ; 30.3 \pm 1.8 ; 49\end{array}$ & $\begin{array}{l}\text { Gp1: Fortified HM }<50 \% \\
\text { intake; MOM + DHM + } \\
\text { BovF + PTF }(34.9 \% \pm 12.5) \\
\text { Gp2: Fortified HM } \geq 50 \% \\
\text { intake; MOM + DHM + } \\
\text { BovF + PTF }(80.9 \% \pm 15.5)\end{array}$ & C3: Gp2 vs. Gp1 & $\begin{array}{l}\text { Hospital stay } \\
\text { Targeted fortification } \\
\text { commenced when } \\
\text { intake } \geq 80 \mathrm{~mL} / \mathrm{kg}\end{array}$ & $\begin{array}{l}\text { Growth (from birth to } \\
\text { TCA): wt z-score SE, wt } \\
\text { gain g/kg/day } \\
\text { Body composition (ADP } \\
\text { at TCA); FM (\%), FFM } \\
(\%)\end{array}$ & Low \\
\hline $\begin{array}{l}\text { Schanler } 1999 \text { [9] } \\
\text { USA }\end{array}$ & $\begin{array}{l}\text { Secondary } \\
\text { analysis of RCT }\end{array}$ & $\begin{array}{l}108 \text { infants } \\
\text { Gp1: } 1069 \pm 169 ; 27.9 \pm 1.2 ; 62 \\
\text { Gp2: } 1044 \pm 185 ; 27.9 \pm 1.1 ; 46\end{array}$ & $\begin{array}{l}\text { Gp1: Fortified HM; MOM + } \\
\text { BovF }(84 \pm 20 \% \text {, median } \\
\text { 93\%) } \\
\text { Gp2: EPTF }(0 \%)\end{array}$ & $\begin{array}{l}\text { C2: Gp1 vs. Gp2 } \\
\text { Knee-heel length synthesised } \\
\text { narratively }\end{array}$ & Hospital stay & $\begin{array}{l}\text { Growth (from } \\
\text { minimum wt to DC): wt } \\
\text { gain }(\mathrm{g} / \mathrm{kg} / \mathrm{d}), \mathrm{HC} \text { gain } \\
(\mathrm{cm} / \mathrm{wk}) \text {, length gain } \\
(\mathrm{cm} / \mathrm{wk})\end{array}$ & Low \\
\hline
\end{tabular}


Table 1. Cont.

\begin{tabular}{|c|c|c|c|c|c|c|c|}
\hline $\begin{array}{l}\text { Study } \\
\text { Country }\end{array}$ & Design & $\begin{array}{l}\text { Participants } \\
\text { BW, g; GA, wk; } n\end{array}$ & $\begin{array}{l}\text { Intervention } \\
\text { (Proportion of HM \% } \\
\text { Unless Stated Elsewhere) }\end{array}$ & $\begin{array}{l}\text { Comparisons for This } \\
\text { Review } \\
\text { C1: EHM vs. EPTF } \\
\text { C2: Any HM vs. EPTF } \\
\text { C3: High- vs. Low-Dose HM }\end{array}$ & Study Duration & Outcomes & Risk of Bias \\
\hline $\begin{array}{l}\text { Sisk } 2008 \text { [60] } \\
\text { USA }\end{array}$ & Cohort & $\begin{array}{l}127 \text { infants } \\
\text { Gp1: } 978 \pm 149 ; 27.8 \pm 2.1 ; 34 \\
\text { Gp2: } 1000 \pm 149 ; 27.4 \pm 1.6 ; 93\end{array}$ & $\begin{array}{l}\text { Gp1: Lower HM }(<50 \% \text { of } \\
\text { HM) } \\
\text { Gp2: Higher HM }(\geq 50 \% \text { of } \\
\text { HM) }\end{array}$ & C3: Gp 1 vs. Gp 2 & Hospital stay & $\begin{array}{l}\text { Growth (from regained } \\
\text { BW to DC): wt gain } \\
\text { (g/kg/day) (reported as } \\
\text { median, IQR, and } \\
\text { converted to mean, SD) }\end{array}$ & Low \\
\hline $\begin{array}{l}\text { Sisk } 2017 \text { [61] } \\
\text { USA }\end{array}$ & Cohort & $\begin{array}{l}551 \text { infants } \\
\text { Gp1: } 1017 \pm 291 ; 27.8 \pm 2.4 ; \\
\text { 299 } \\
\text { Gp2: } 1026 \pm 270 ; 28.0 \pm 2.4 ; \\
\text { 139 } \\
\text { Gp3: } 1036 \pm 21 ; 28.1 \pm 2.6 ; 113\end{array}$ & $\begin{array}{l}\text { Gp1: MOM; } \geq 50 \% \text { MOM } \\
\text { (MOM 97\%) } \\
\text { Gp2: PDHM; } \geq 50 \% \text { PDHM } \\
\text { (PDHM 86\%) } \\
\text { Gp3: PTF; } \geq 50 \% \text { PTF (PTF } \\
\text { 91\%) }\end{array}$ & C3: Gp1 + Gp2 vs. Gp3 & $\begin{array}{l}\text { SS: birth } \\
\text { SE: NEC diagnosis or } \\
34 \text { wk PMA }\end{array}$ & $\begin{array}{l}\text { Growth (from birth to } \\
\text { DC): wt gain } \\
\text { (g/kg/day), HC gain } \\
\text { (cm/wk), length gain } \\
\text { (cm/wk), change in } \\
\text { z-scores (reported as } \\
\text { median, IQR and } \\
\text { converted to mean, SD) }\end{array}$ & Low \\
\hline $\begin{array}{l}\text { Soldateli } 2020 \text { [62] } \\
\text { USA }\end{array}$ & $\begin{array}{l}\text { Cohort } \\
\text { (secondary } \\
\text { analysis of data } \\
\text { collected for QI } \\
\text { initiative) }\end{array}$ & $\begin{array}{l}1429 \text { infants } \\
\text { Whole cohort: } 1080(861,1285) \text {; } \\
28(27,30)\end{array}$ & $\begin{array}{l}\text { Gp1: } 0-25 \% \mathrm{HM} \\
\text { Gp2: } 26-50 \% \mathrm{HM} \\
\text { Gp3: } 51-75 \% \mathrm{HM} \\
\text { Gp4: } 76-99 \% \\
\text { Gp5: } 100 \% \mathrm{HM} \\
\text { Diet recorded on days of } \\
\text { life } 7,14,21,28,42,56,70, \\
\text { 84, and at discharge or } \\
\text { transfer }\end{array}$ & C3: synthesised narratively & Birth to DC or transfer & $\begin{array}{l}\text { Growth (from birth to } \\
\text { DC or transfer): wt gain } \\
\text { (g/kg/day), change in } \\
\text { wt and length z-scores } \\
\text { (only mean reported in } \\
\text { text) }\end{array}$ & $\begin{array}{l}\text { Moderate (unable } \\
\text { to determine if } \\
\text { Gps comparable) }\end{array}$ \\
\hline
\end{tabular}


Table 1. Cont.

\begin{tabular}{|c|c|c|c|c|c|c|c|}
\hline $\begin{array}{l}\text { Study } \\
\text { Country }\end{array}$ & Design & $\begin{array}{l}\text { Participants } \\
\text { BW, g; GA, wk; } n\end{array}$ & $\begin{array}{l}\text { Intervention } \\
\text { (Proportion of HM \% } \\
\text { Unless Stated Elsewhere) }\end{array}$ & $\begin{array}{l}\text { Comparisons for This } \\
\text { Review } \\
\text { C1: EHM vs. EPTF } \\
\text { C2: Any HM vs. EPTF } \\
\text { C3: High- vs. Low-Dose HM }\end{array}$ & Study Duration & Outcomes & Risk of Bias \\
\hline $\begin{array}{l}\text { Spielger } 2016 \text { [63] } \\
\text { Germany }\end{array}$ & Cohort & $\begin{array}{l}1433 \text { infants } \\
\text { Gp1: } 1080 \text { (830, 1330); } 28.7 \\
(26.6,30.1) ; 239 \\
\text { Gp2: } 1100(865,1340) ; 29.0 \\
(26.9,30.0) ; 223 \\
\text { Gp3: } 1050(805,1295) ; 28.4 \\
(26.6,30.0) ; 971\end{array}$ & $\begin{array}{l}\text { Gp1: EPTF; PTF (0\%) } \\
\text { Gp2: EHM; MOM + DHM } \\
\text { (100\%) } \\
\text { Gp3: Mixed; MOM + DHM + } \\
\text { PTF (NR) }\end{array}$ & $\begin{array}{l}\text { C1: Gp1 vs. Gp2 } \\
\text { C2: Gp2 + Gp3 vs. Gp1 } \\
\text { C3: Gp2 vs. Gp1 + Gp2 }\end{array}$ & Hospital stay & $\begin{array}{l}\text { Growth (from birth to DC): } \\
\text { change in wt z-score, } \\
\text { (reported as median, IQR, } \\
\text { and converted to mean, } \\
\text { SD) }\end{array}$ & Low \\
\hline $\begin{array}{l}\text { Verd } 2015[42] \\
\text { Spain }\end{array}$ & $\begin{array}{l}\text { Interrupted time } \\
\text { series }\end{array}$ & $\begin{array}{l}201 \text { infants } \\
\text { Gp1: } 800(410,995) ; \text { GA (d) } \\
\text { 185 (161, 236); } 148 \\
\text { Gp2: } 830(440,998) ; 190 \text { (166, } \\
\text { 239); } 53\end{array}$ & $\begin{array}{l}\text { Gp1: EHM; MOM + DHM } \\
(100 \%) \\
\text { Gp2: Any PTF; MOM + } \\
\text { DHM + PTF (NR) }\end{array}$ & C3: Gp1 vs. Gp2 & Hospital stay & $\begin{array}{l}\text { Growth (from birth to DC): } \\
\text { change in wt, length, and } \\
\text { HC z-scores reported as } \\
\text { median, IQR, and } \\
\text { converted to mean, SD }\end{array}$ & Low \\
\hline $\begin{array}{l}\text { Warner } 1998 \text { [64] } \\
\text { United Kingdom }\end{array}$ & Cohort & $\begin{array}{l}59 \text { infants, median(range) } \\
\text { Gp1: } 1178(685,1510) ; 29.6(25, \\
\text { 33); } 38 \\
\text { Gp2: } 1120(840,1580) ; 29.1(25, \\
34) ; 21\end{array}$ & $\begin{array}{l}\text { Gp1: HM; MOM + BovF + } \\
\text { (term infant formula if MOM } \\
\text { was insufficient) (NR) } \\
\text { Gp2: EPTF }(0 \%)\end{array}$ & C2: Gp1 vs. Gp2 & $\begin{array}{l}\text { SS: at birth } \\
\text { SE: wt of } 1800 \mathrm{~g} \text { was } \\
\text { reached }\end{array}$ & $\begin{array}{l}\text { Growth: wt gain (from } \\
\text { start of full feeds to } 1800 \mathrm{~g} \text {, } \\
\mathrm{g} / \mathrm{kg} / \mathrm{d} \text { ), HC gain (from SS } \\
\text { to } \mathrm{SE}, \mathrm{cm} / \mathrm{wk} \text { ), length gain } \\
\text { (from } \mathrm{SS} \text { to } \mathrm{SE}, \mathrm{cm} / \mathrm{wk} \text { ) }\end{array}$ & Low \\
\hline $\begin{array}{l}\text { Wauben } 1998 \text { [21,65] } \\
\text { Canada }\end{array}$ & $\begin{array}{l}\text { RCT with } \\
\text { non-randomised } \\
\text { reference group }\end{array}$ & $\begin{array}{l}37 \text { infants } \\
\text { Gp1: } 1400 \pm 200 ; 29.9 \pm 1.9 ; 12 \\
\text { Gp2: } 1300 \pm 200 ; 30.1 \pm 1.5 ; 13 \\
\text { Gp3: } 1200 \pm 200 ; 29.7 \pm 1.7,12\end{array}$ & $\begin{array}{l}\text { Gp1: MOM + multinutrient } \\
\text { fortifier }(100 \%) \\
\text { Gp2: MOM + calcium and } \\
\text { phosphorus alone }(100 \%) \\
\text { Gp3: PTF }(0 \%) \text { (comparison } \\
\text { group, not randomised) }\end{array}$ & $\begin{array}{l}\text { C1: Gp1 vs. Gp3 } \\
\text { Body composition C3: Gp1 vs. } \\
\text { Gp3 } \\
\text { (Gp2 not fortified therefore } \\
\text { excluded) }\end{array}$ & $\begin{array}{l}\text { SS: full oral feeds } \geq 5 \mathrm{~d} \\
\text { SE: Discharge home or } \\
>38 \text { wk PMA } \\
\text { (whichever came first) }\end{array}$ & $\begin{array}{l}\text { Growth (from SS to DC): } \\
\text { wt gain }(\mathrm{g} / \mathrm{kg} / \mathrm{d}), \mathrm{HC} \text { gain } \\
(\mathrm{cm} / \mathrm{wk}), \text { length gain } \\
(\mathrm{cm} / \mathrm{wk})[21] \\
\text { Body composition (DXA) } \\
\text { at TCA: FM }(\%), \text { FFM }(\%) \\
\text { [65] }\end{array}$ & Low \\
\hline
\end{tabular}

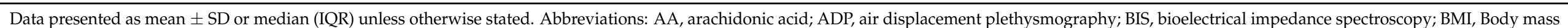

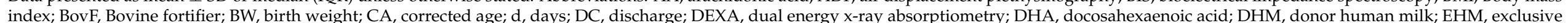

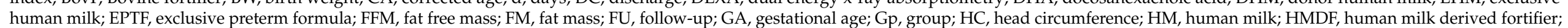

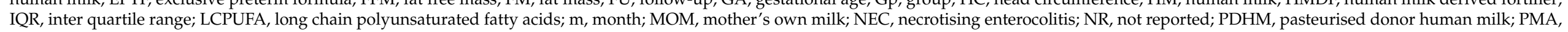

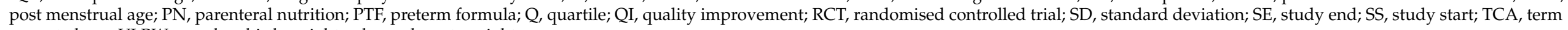
corrected age; VLBW, very low birth weight; wk, weeks; wt, weight. 
Table 2. Summary of findings.

\begin{tabular}{ll}
\hline & EPTF vs. EHM \\
Anticipated Absolute Effects MD (95\% CI); \\
Outcome & Garticipants; $N$ Studies \\
& GRADE Certainty of Evidence \\
Interpretation
\end{tabular}

Randomised controlled trial

MD 2 ( -1.54 to 5.54$) ; n=53 ; 1$ study

Interpretation: Inconclusive

WEIGHT GAIN, G/DAY

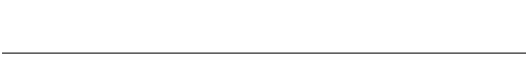

\section{WEIGHT GAIN, G/KG/DAY}

Certainty: not graded; $\mathrm{ROB}$ moderate

EPTF vs. Any HM
Anticipated Absolute Effects MD $(95 \% \mathrm{CI})$;
$N$ Participants; $N$ Studies
GRADE Certainty of Evidence
Interpretation

Interpretation
Observational studies

MD 2.03 ( -0.31 to 4.38$) ; n=364 ; 4$ studies

Certainty: very low

Interpretation: Inconclusive
Observational studies

MD 1.97 (0.21 to 3.72$) ; n=795 ; 5$ studies

Certainty: very low

Interpretation: Inconclusive

\section{Low- vs. High-Dose HM \\ Anticipated Absolute Effects MD ( $95 \% \mathrm{CI})$; \\ $N$ Participants; $N$ Studies \\ GRADE Certainty of Evidence}

Interpretation

Observational studies

MD $-0.83(-1.65$ to 0$) ; n=1606 ; 2$ studies

Certainty: low

Interpretation: Possible effect of a small decrease in weight gain ( $\mathrm{g} /$ day) with lower-dose HM versus

higher-dose HM

\section{Randomised controlled trial}

MD 2.41 (1.09 to 3.72$) ; n=373 ; 2$ studies

Certainty: low

Observational studies

MD 0.56 ( 0.09 to 1.03$) ; n=3162 ; 13$ studies

Certainty: very low

Interpretation: Possible effect of a small increase in

weight gain $(\mathrm{g} / \mathrm{kg} /$ day) with lower-dose HM versus high-dose HM

Randomised controlled trial

MD 0 ( -0.29 to 0.29$) ; n=326 ; 1$ study

Certainty: not graded; ROB low

Observational studies

MD 0.26 ( 0.03 to 0.48$) ; n=49 ; 2$ studies

Certainty: low

Observational studies

MD 0.21 ( -0.15 to 0.56$) ; n=1532 ; 3$ studies

Interpretation: Possible effect of a small increase in Certainty: very low

Observational studies

Interpretation: Inconclusive

MD 0.19 ( 0.6 to 0.33$) ; n=4059 ; 12$ studie

Certainty: very low

Interpretation: Inconclusive

Randomised controlled trial

Randomised controlled trial

MD 0.1 ( -0.02 to 0.22$) ; n=53 ; 1$ study

Observational studies

MD $0(-0.06$ to 0.06$) ; n=373 ; 2$ studie

Certainty: moderate

Observational Studies

Certainty: not graded; $\mathrm{ROB}$ moderate

MD 0.06 ( 0.1 to 0.11$) ; n=495 ; 4$ studies

Observational studies

Certainty: low

MD 0.09 ( -0.10 to 0.29$) ; n=78 ; 2$ studies

Interpretation:

gain in ins

Interpretation: Inconclusive
Certainty: very low

Interpretation: Possibly no effect of $\mathrm{HM}$ dose on $\mathrm{HC}$ gain $(\mathrm{cm} / \mathrm{wk})$ 
Table 2. Cont

\begin{tabular}{|c|c|c|c|}
\hline Outcome & $\begin{array}{l}\text { EPTF vs. EHM } \\
\text { Anticipated Absolute Effects MD }(95 \% \mathrm{CI}) ; \\
N \text { Participants; } N \text { Studies } \\
\text { GRADE Certainty of Evidence } \\
\text { Interpretation }\end{array}$ & $\begin{array}{l}\text { EPTF vs. Any HM } \\
\text { Anticipated Absolute Effects MD }(95 \% \mathrm{CI}) ; \\
N \text { Participants; } N \text { Studies } \\
\text { GRADE Certainty of Evidence } \\
\text { Interpretation }\end{array}$ & $\begin{array}{l}\text { Low- vs. High-Dose HM } \\
\text { Anticipated Absolute Effects MD }(95 \% \mathrm{CI}) ; \\
N \text { Participants; } N \text { Studies } \\
\text { GRADE Certainty of Evidence } \\
\text { Interpretation }\end{array}$ \\
\hline CHANGE IN HC Z-SCORE & $\begin{array}{l}\text { Observational study } \\
\text { MD } 0.1(-0.42 \text { to } 0.62) ; n=32,1 \text { study } \\
\text { Certainty: not graded; ROB low } \\
\text { Interpretation: Inconclusive }\end{array}$ & $\begin{array}{l}\text { Observational studies } \\
\text { MD } 0.43(0.18 \text { to } 0.69) ; n=322 ; 2 \text { studies } \\
\text { Certainty: low } \\
\text { Interpretation: Possible effect of small increase in HC } \\
\text { z-score with EPTF versus any HM. }\end{array}$ & $\begin{array}{l}\text { Randomised controlled trial } \\
\text { MD } 0.2(-0.08 \text { to } 0.48) ; n=326 ; 1 \text { study } \\
\text { Certainty: not graded; ROB low } \\
\text { Observational studies } \\
\text { MD } 0.09 \text { ( }-0.19 \text { to } 0.38) ; n=2627 ; 8 \text { studies } \\
\text { Certainty: very low } \\
\text { Interpretation: Inconclusive }\end{array}$ \\
\hline LENGTH GAIN, CM/WK & $\begin{array}{l}\text { Randomised controlled trial } \\
\text { MD } 0.28 \text { ( } 0.14 \text { to } 0.42) ; n=53 ; 1 \text { study } \\
\text { Certainty: not graded; ROB moderate } \\
\text { Observational studies } \\
\text { MD } 0.06 \text { ( }-0.07 \text { to } 0.19) ; n=78 ; 2 \text { studies } \\
\text { Certainty: very low } \\
\text { Interpretation: Inconclusive }\end{array}$ & $\begin{array}{l}\text { Observational studies } \\
\text { MD } 0.09 \text { ( }-0.05 \text { to } 0.22) ; n=778 ; 3 \text { studies } \\
\text { Certainty: very low } \\
\text { Interpretation: Inconclusive }\end{array}$ & $\begin{array}{l}\text { Randomised controlled trial } \\
\text { MD - } 0.04 \text { ( }-0.28 \text { to } 0.21) ; n=373 ; 2 \text { studies } \\
\text { Certainty: low } \\
\text { Observational studies } \\
\text { MD } 0.05 \text { ( } 0.02 \text { to } 0.08) ; n=2423 ; 8 \text { studies } \\
\text { Certainty: low } \\
\text { Interpretation: Possibly no effect of dose of human milk } \\
\text { on length gain (cm/wk) }\end{array}$ \\
\hline CHANGE IN LENGTH Z-SCORE & $\begin{array}{l}\text { Observational study } \\
\text { MD } 0.0 \text { ( }-0.63 \text { to } 0.63) ; n=32,1 \text { study } \\
\text { Certainty: not graded; ROB low } \\
\text { Interpretation: Inconclusive }\end{array}$ & No studies detected & $\begin{array}{l}\text { Randomised controlled trial } \\
\text { MD 0.1 ( }-0.26 \text { to } 0.46) ; n=326 ; 1 \text { study } \\
\text { Certainty: not graded; ROB low } \\
\text { Observational study } \\
\text { MD } 0.09 \text { ( }-0.07 \text { to } 0.25) ; n=1131,3 \text { studies } \\
\text { Certainty: very low } \\
\text { Interpretation: Inconclusive }\end{array}$ \\
\hline FAT FREE MASS \% & $\begin{array}{l}\text { Observational studies } \\
\text { MD }-1.46 \text { ( }-4.35 \text { to } 1.43) ; n=87 ; 3 \text { studies } \\
\text { Certainty: very low } \\
\text { Interpretation: Inconclusive }\end{array}$ & No studies identified & $\begin{array}{l}\text { Observational studies } \\
\text { MD }-5.1 \text { ( }-12.45 \text { to } 2.25) ; n=73 ; 1 \text { study } \\
\text { Certainty: not graded; ROB low } \\
\text { Interpretation: Inconclusive }\end{array}$ \\
\hline FAT FREE MASS G & $\begin{array}{l}\text { Observational studies } \\
\text { MD } 130.18 \text { ( } 53.86 \text { to } 206.5) ; n=134 ; 4 \text { studies } \\
\text { Certainty: very low } \\
\text { Interpretation: Inconclusive }\end{array}$ & No studies identified & No studies identified \\
\hline
\end{tabular}


Table 2. Cont.

\section{EPTF vs. EHM}

Anticipated Absolute Effects MD (95\% CI);

$N$ Participants; $N$ Studies

GRADE Certainty of Evidence

Interpretation

Observational studies

MD $1.82(-0.59$ to 4.23$) ; n=141 ; 4$ studies

Certainty: very low

Interpretation: Inconclusive

Observational studies

MD 60.94 ( -5.42 to 127.31$) ; n=134 ; 4$ studies

Certainty: very low
Interpretation: Inconclusive
EPTF vs. Any HM

Anticipated Absolute Effects MD (95\% CI);

$N$ Participants; $N$ Studies

GRADE Certainty of Evidence

Interpretation

No studies identified

No studies identified
Low- vs. High-Dose HM

Anticipated Absolute Effects MD (95\% CI);

$N$ Participants; $N$ Studies

GRADE Certainty of Evidence

Interpretation

Observational studies

MD -0.48 ( -1.7 to 0.73$) ; n=133 ; 1$ study

Certainty: not graded; ROB low

Interpretation: Inconclusive

\section{FAT MASS G}

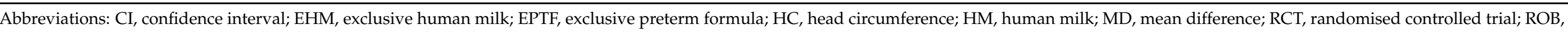

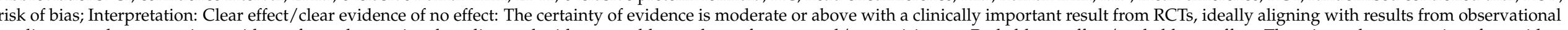

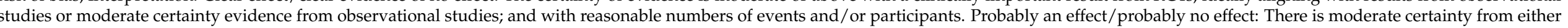

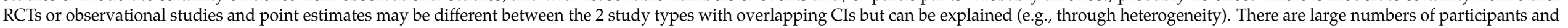

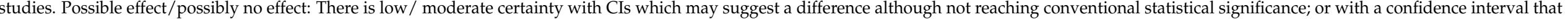
indicates a trivial difference only. Inconclusive: The certainty of evidence is very low to low, CIs are wide, and number of participants and studies is low. 
Observational studies: Four studies reporting $\mathrm{g} / \mathrm{kg} / \mathrm{d}$ were included in the metaanalysis: $[21,35,39,57]$. There was no clear difference in the rate of weight gain between groups (MD 2.03, 95\% CI -0.31 to $4.38, n=364, \mathrm{I}^{2}=87 \%$, Figure 2, very low certainty evidence, Table S3). The rate of weight gain was assessed across different time periods between studies, including from birth to discharge [39], full oral feeds tolerated for $\geq 5$ days to discharge [21], enteral feed volume $\geq 150 \mathrm{~mL} / \mathrm{kg} / \mathrm{d}$ to nasogastric feeds no longer required [35], and full enteral feeding to discharge [57].

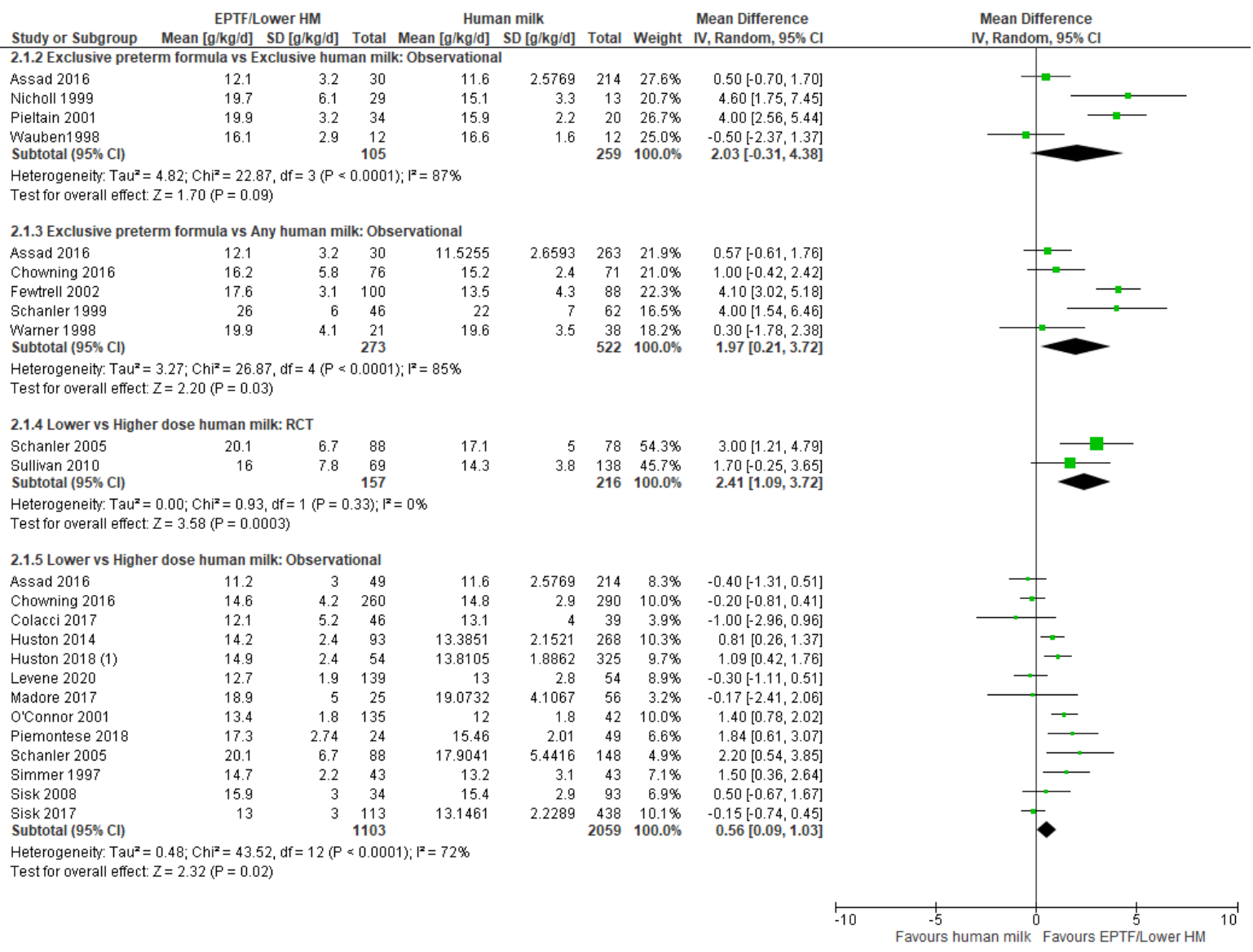

Footnotes

(1) Some overlap of infants with Huston 2014 but exclusion of this study did not change the result

Figure 2. Forest plot of mean difference in weight gain $(\mathrm{g} / \mathrm{kg} /$ day) and human milk intake.

An additional two studies $[45,53]$ were unable to be included in the meta-analysis. Carlson 1998 [45] reported weight gain $(\mathrm{g} / \mathrm{kg} / \mathrm{d})$ according to different stages of the hospital admission and found higher weight gain with EPTF versus EHM fed infants over the time periods 15-35 days and 57 days to term (Table S1). In contrast, Manea 2016 [53] reported greater weight gain $(\mathrm{g} / \mathrm{d})$ in the EHM group during the first five weeks of life (Table S1).

Two studies reported change in z-scores $[55,63]$ with EPTF-fed infants having a greater increase in z-scores than EHM-fed infants (MD 0.26, 95\% CI 0.03 to $0.48, n=494, \mathrm{I}^{2}=26 \%$, Figure 3, low certainty evidence, Table S4). 


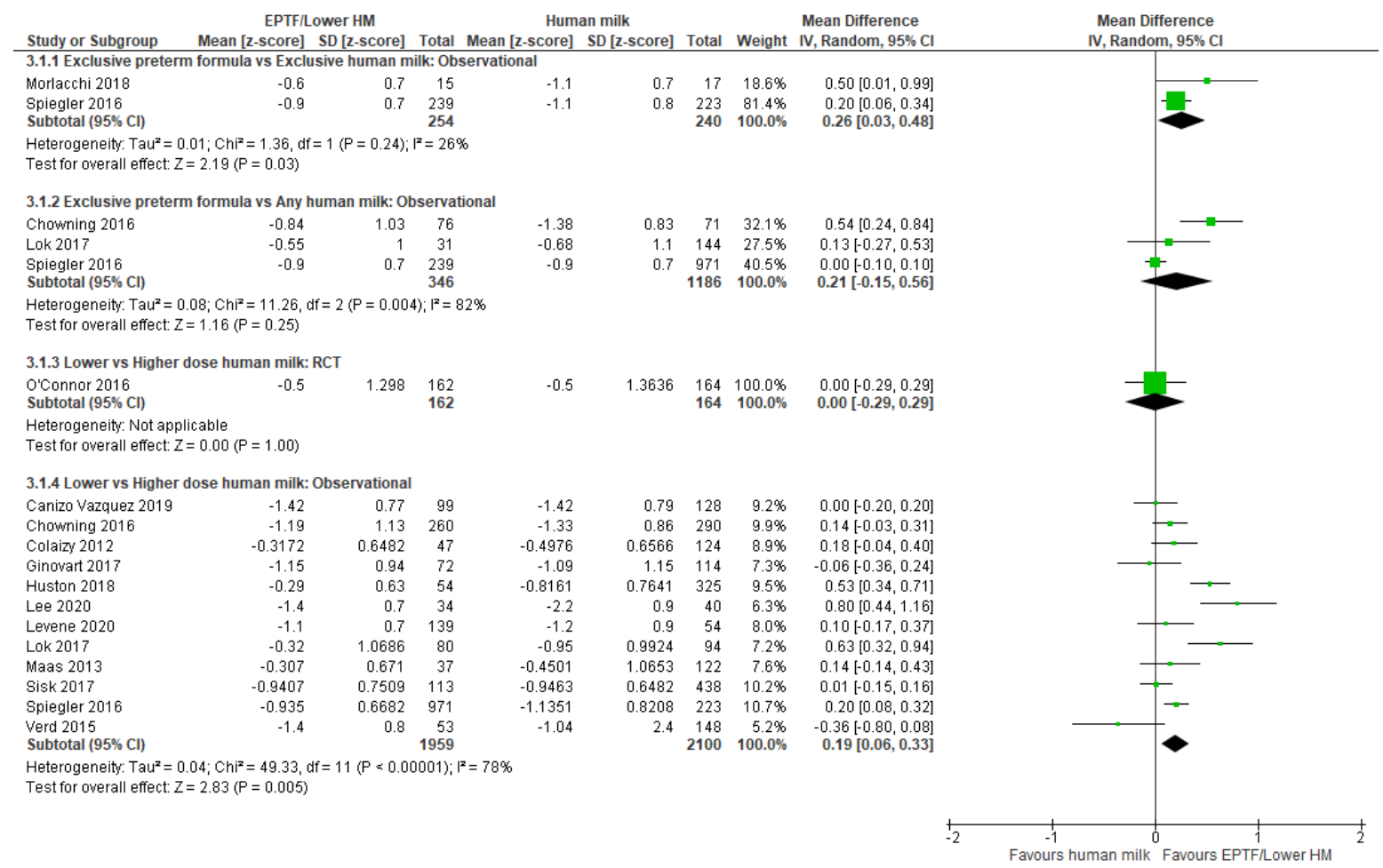

Figure 3. Forest plot of mean difference in change in weight z-scores and human milk intake.

Overall: The evidence for an effect of EPTF vs. EHM feeding on weight gain is inconclusive.

\subsubsection{Comparison 2: Exclusive Preterm Formula vs. Any Human Milk}

Randomised trials: There were no RCTs reporting weight gain for this comparison.

Observational studies: Six studies reported weight gain in $\mathrm{g} / \mathrm{kg} / \mathrm{d}$ with five included in the meta-analysis $[9,19,39,47,64]$. EPTF-fed infants had a higher rate of weight gain (MD $1.97,95 \%$ CI 0.21 to $3.72, n=795, \mathrm{I}^{2}=85 \%$, Figure 2, very low certainty evidence, Table S3). Heterogeneity may be explained by baseline difference in the study duration and varying intake of HM. Carlson 1998 [45] assessed weight gain over different time periods and showed higher weight gain $(\mathrm{g} / \mathrm{kg} / \mathrm{d})$ in infants receiving EPTF compared with any HM over the time period 15-35 days and 57 days to term (Table S1).

Change in z-scores was reported in three studies $[22,47,63]$ and all were included in a meta-analysis. There was no clear difference in change in z-score between infants receiving EPTF and any HM (MD 0.21, 95\% CI 0.15 to $0.56, n=1532, \mathrm{I}^{2}=82 \%$, Figure 3 , very low certainty evidence, Table S4). Heterogeneity may be due to the varying doses of HM (Table 1).

Overall: The evidence for an effect of EPTF vs. any HM feeding on weight gain is inconclusive.

\subsubsection{Comparison 3: Lower- vs. Higher-Dose Human Milk}

Randomised Trials: Meta-analysis of two RCTs [33,34] showed a higher rate of weight gain $\left(\mathrm{g} / \mathrm{kg} / \mathrm{d}\right.$ ) in the lower-dose HM group (MD 2.41, 95\%CI 1.09 to $3.72, n=373, \mathrm{I}^{2}=0 \%$, Figure 2, low certainty evidence, Table S3). O'Connor 2016 [32] showed no clear difference between lower- and higher-dose HM intake on change in weight z-score (MD 0.0, 95\% CI -0.29 to $0.29, n=326$, Figure 3, low risk of bias, Table S4).

Observational studies: Two studies reported weight gain in $\mathrm{g} / \mathrm{d}$ and were included in a meta-analysis $[36,48]$ with a possible difference between lower and higher doses of $\mathrm{HM}\left(\mathrm{MD}-0.83,95 \% \mathrm{CI}-1.65\right.$ to $0.00, n=1606, \mathrm{I}^{2}=0 \%$, Figure 1 , low certainty evidence, Table S2). 
Thirteen studies were included in the meta-analysis for weight gain $(\mathrm{g} / \mathrm{kg} / \mathrm{d})$ for this comparison $[20,28,29,33,39,40,47,50,52,58-61]$. Lower doses of HM were associated with a higher rate of weight gain $(\mathrm{g} / \mathrm{kg} / \mathrm{d})\left(\mathrm{MD} 0.56,95 \%\right.$ CI 0.09 to $1.03, n=3162, \mathrm{I}^{2}=72 \%$, Figure 2, very low certainty evidence, Table S3). Heterogeneity is possibly due to differences in study design and varying dosage of HM. Also of note is that Sisk 2017 [61] used both a multi-nutrient and protein fortifier with the aim of providing a protein intake of $4 \mathrm{~g} / \mathrm{kg} / \mathrm{d}$.

Two studies [43,62] reported weight gain as $\mathrm{g} / \mathrm{kg} / \mathrm{d}$ but were not included in the meta-analysis, as they did not directly compare groups [43] or did not report SDs [62] (Table S1). Brownell 2018 [43] showed that the mean growth rate decreased by $0.17 \mathrm{~g} / \mathrm{kg} / \mathrm{d}$ for every $10 \%$ increase in DHM intake but did not vary with PTF intake (using MOM as reference) (Table S1), whereas Soldateli 2020 [62] reported no difference in growth velocity $(\mathrm{g} / \mathrm{kg} / \mathrm{d})$ across five categories of HM intake $(p=0.3)$ or between the lowest category $(0-25 \% \mathrm{HM})$ and the highest category $(100 \% \mathrm{HM})(p=0.6)$ (Table S1).

Seventeen studies reported weight gain as change in z-scores during the hospital admission, and 12 of these were included in the meta-analysis [7,8,22,28,42,44,47,49-51,61,63]. Infants fed lower-dose $\mathrm{HM}$ had a greater increase in z-score change than those fed higherdose HM (MD 0.19, 95\% CI 0.06 to $0.33, n=4059, \mathrm{I}^{2}=78 \%$, Figure 3, very low certainty evidence, Table S4). Heterogeneity is possibly due to difference in doses of HM and differences in type of fortifier used. Five studies $[38,41,43,46,62]$ were not included in a meta-analysis. Soldateli 2020 [62] showed no difference in change in weight z-score across five categories of HM intake ( $p=0.7)$ or between the lowest category $(0-25 \% \mathrm{HM})$ and the highest category $(100 \% \mathrm{HM})(p=0.2)$ (Table S1). The remaining four studies $[38,41,43,46]$ all reported a greater increase in weight z-score associated with lower doses of HM (Table S1).

Overall: There is a possible effect that lower doses of HM compared with higher doses of HM improve weight gain during the hospital admission.

\subsection{Head Circumference Gain and Human Milk Feeding}

Thirty studies reported HC gain. Nineteen studies reported HC growth in $\mathrm{cm} /$ wk $[9,19-21,28,29,31,33,34,43,47,48,52,56,57,59,61,62,64]$, and 16 reported change in z-scores $[8,22,28,32,38,41,43,44,46,47,50,51,55,61,62,64]$.

\subsubsection{Comparison 1: Exclusive Preterm Formula vs. Exclusive Human Milk}

Randomised trials: There was no clear difference in HC growth $(\mathrm{cm} / \mathrm{wk})$ in the one RCT [31] reporting this comparison (MD $0.10,95 \%$ CI -0.02 to $0.22, n=53$, Figure 4 , moderate risk of bias, Table S5).

Observational studies: Meta-analysis of two studies $[21,57]$ showed no clear difference in HC gain (cm/wk) (MD 0.09, 95\% CI -0.10 to $0.29, n=78, \mathrm{I}^{2}=84 \%$, Figure 4, very low certainty evidence, Table S5). Heterogeneity is likely due to baseline differences in population and study design.

One study reported change in HC z-scores [55] and showed no clear difference between the EPTF and EHM fed groups (MD 0.10, 95\% CI -0.42 to $0.62, n=32$, Figure 5, low risk of bias, Table S6).

Overall: The evidence for an effect of EPTF vs. EHM feeding on HC growth is inconclusive.

\subsubsection{Comparison 2: Exclusive Preterm Formula vs. Any Human Milk}

Randomised trials: There were no RCTs identified reporting HC gain for this comparison.

Observational studies: Four studies $[9,19,47,64]$ reported HC gain $(\mathrm{cm} / \mathrm{wk})$ in infants fed EPTF compared with any HM. On meta-analysis, EPTF-fed infants had a higher rate of HC gain (MD 0.06, 95\% CI 0.01 to $0.11, n=495, \mathrm{I}^{2}=18 \%$, Figure 4, low certainty evidence, Table S5). 


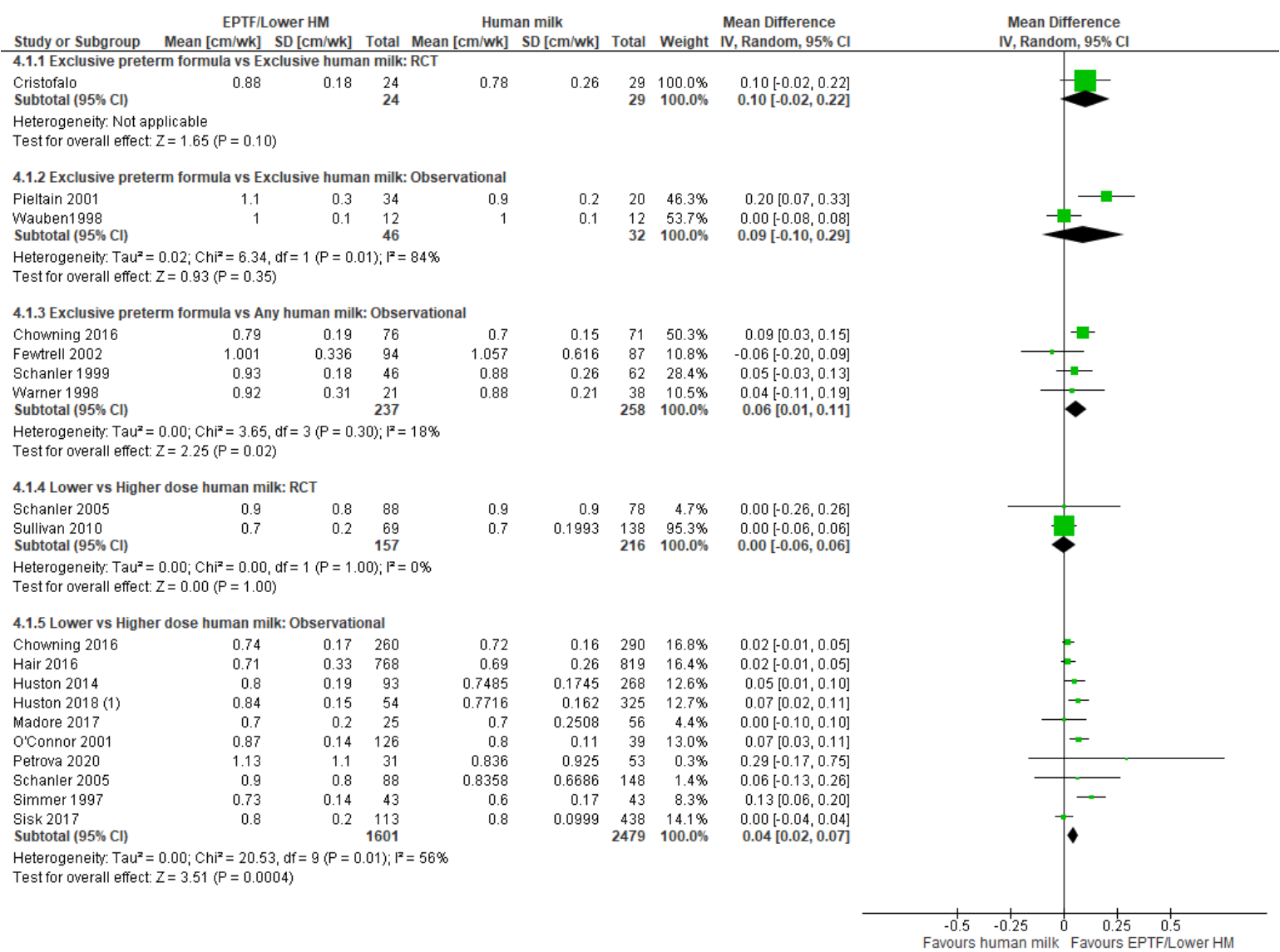

Footnotes

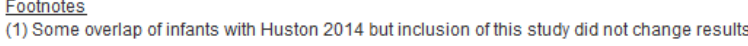

Figure 4. Forest plot of mean difference in change in head circumference gain ( $\mathrm{cm} / \mathrm{wk})$ and human milk intake.

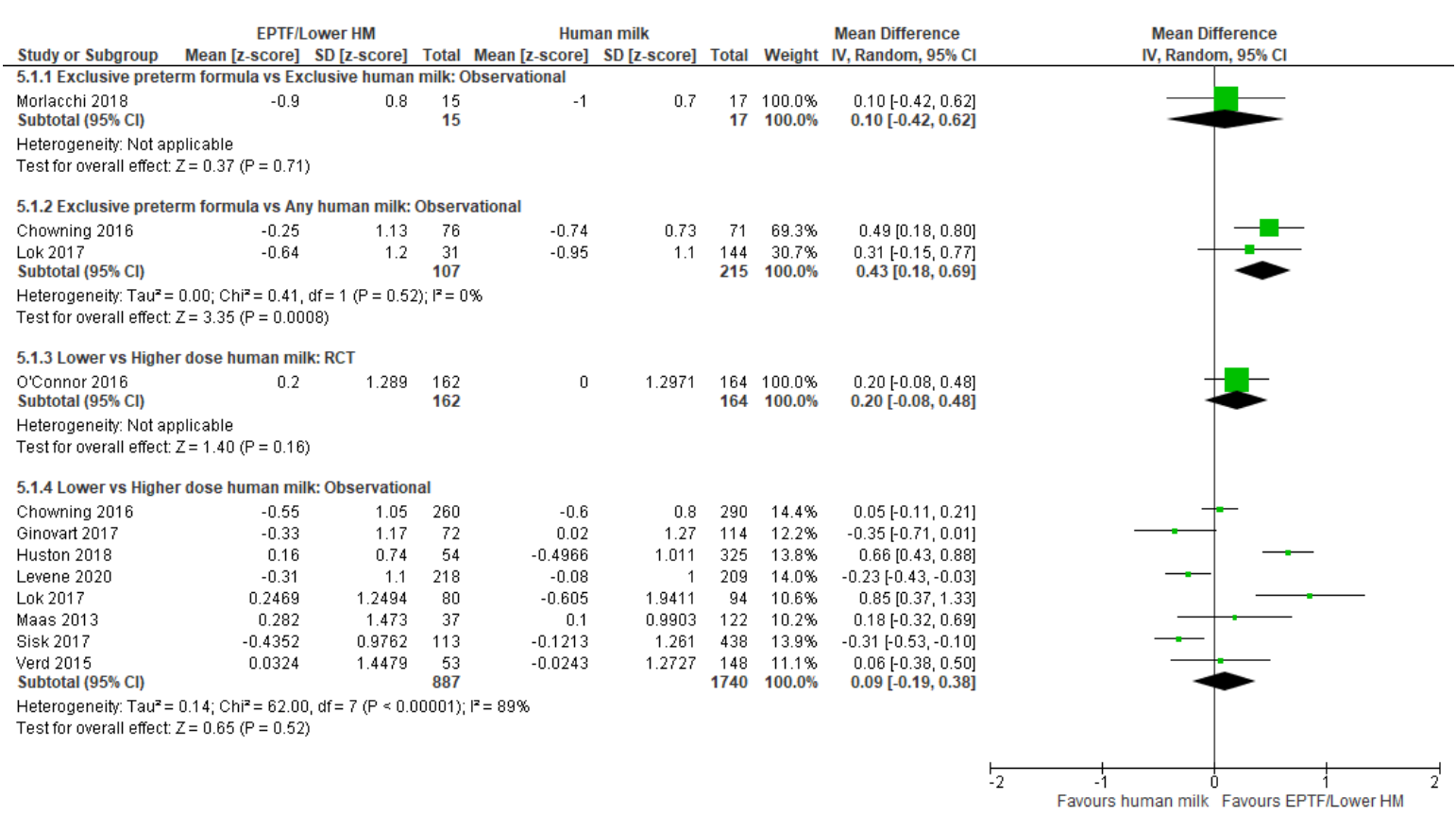

Figure 5. Forest plot of mean difference in change in head circumference z-scores and human milk intake. 
Two studies [22,47] reported change in HC z-score, and on meta-analysis, there was a greater increase in HC z-scores in the infants fed EPTF (MD 0.43, 95\% CI 0.18 to 0.69, $n=322, \mathrm{I}^{2}=0 \%$, Figure 5, low certainty evidence, Table S6).

Overall: There is a possible effect that feeding EPTF compared with any HM is associated with small increases in HC gain during the hospital admission.

\subsubsection{Comparison 3: Lower- vs. Higher-Dose Human Milk}

Randomised trials: There was no clear difference in $\mathrm{HC}$ growth (cm/wk) in the metaanalysis of the two RCTs reporting this outcome [33,34] (MD 0.00, 95\% CI -0.06 to 0.06, $n=373, \mathrm{I}^{2}=0 \%$, Figure 4, moderate certainty evidence, Table S5). One RCT [32] reported change in $\mathrm{HC}$ z-score and showed no difference between groups (MD 0.20, 95\% CI - 0.08 to $0.48, n=326$, Figure 5, low risk of bias, Table S6).

Observational studies: Twelve studies reported HC growth in $\mathrm{cm} /$ wk with 10 included in the meta-analysis $[20,28,29,33,47,48,52,56,59,61]$ showing greater HC gain associated with lower-dose HM (MD $0.04,95 \%$ CI 0.02 to $0.07, n=4080, \mathrm{I}^{2}=56 \%$, Figure 4 , very low certainty evidence, Table S5).

Two studies could not be included in the meta-analysis [43,62]. Brownell 2018 [43] showed that, in reference to MOM, increased DHM intake was associated with decreased HC growth (cm/wk), but PTF was not (Table S1). Soldateli 2020 [62] reported no difference in HC growth (cm/wk) across five categories of HM intake $(p=0.4)$ or between the lowest category (0-25\% HM) and the highest category (100\% HM) $(p=0.1)$ (Table S1).

Thirteen studies reported change in $\mathrm{HC}$ z-scores, with eight included in the metaanalysis $[8,22,28,42,47,50,51,61]$. There was no clear difference in change in $\mathrm{HC}$ z-score between lower and higher-dose HM-fed infants (MD 0.09, 95\% CI -0.19 to 0.38, $n=2627$, $\mathrm{I}^{2}=89 \%$, Figure 5, very low certainty evidence, Table S6). Heterogeneity is likely due to difference in proportion of HM and PTF dosage.

Five studies reported change in $\mathrm{HC}$ z-scores but could not be included in the metaanalysis $[38,41,43,46,62]$. Three studies [38,41,43] compared the difference between a reference, either MOM [41,43] or EHM [38], and found no relationship between formula intake and HC, although Brownell 2018 [43] also found that increased DHM intake was significantly associated with decreased change in HC z-scores (Table S1). Castellano Yanez 2019 [46] reported a greater increase in HC z-scores with lower-dose HM (Table S1). However, Soldateli 2020 [62] reported no difference in change in HC z-score across five categories of HM intake $(p=0.8)$ or between the lowest category $(0-25 \% \mathrm{HM})$ and the highest category $(100 \% \mathrm{HM})(p=0.2)$ (Table S1).

Overall: There is possibly no effect of lower vs. higher doses of HM feeding on HC growth.

\subsection{Length Gain and Human Milk Feeding}

Twenty-five studies reported length gain in a variety of ways: 15 as cm/wk [9,20, $21,28,29,31,33,34,37,43,52,57,59,61,64], 11$ as change in $z$-score $[28,32,38,41-44,46,55,61,62]$, and two studies reported lower leg growth $[35,36]$.

\subsubsection{Comparison 1: Exclusive Preterm Formula vs. Exclusive Human Milk}

Randomised trials: One small RCT [31] reported the effect of EPTF feeding compared with EHM feeding on linear growth and found a higher length gain $(\mathrm{cm} / \mathrm{wk})$ with EPTF (MD 0.28, 95\% CI 0.14 to $0.42, n=53$, Figure 6, moderate risk of bias, Table S7). 


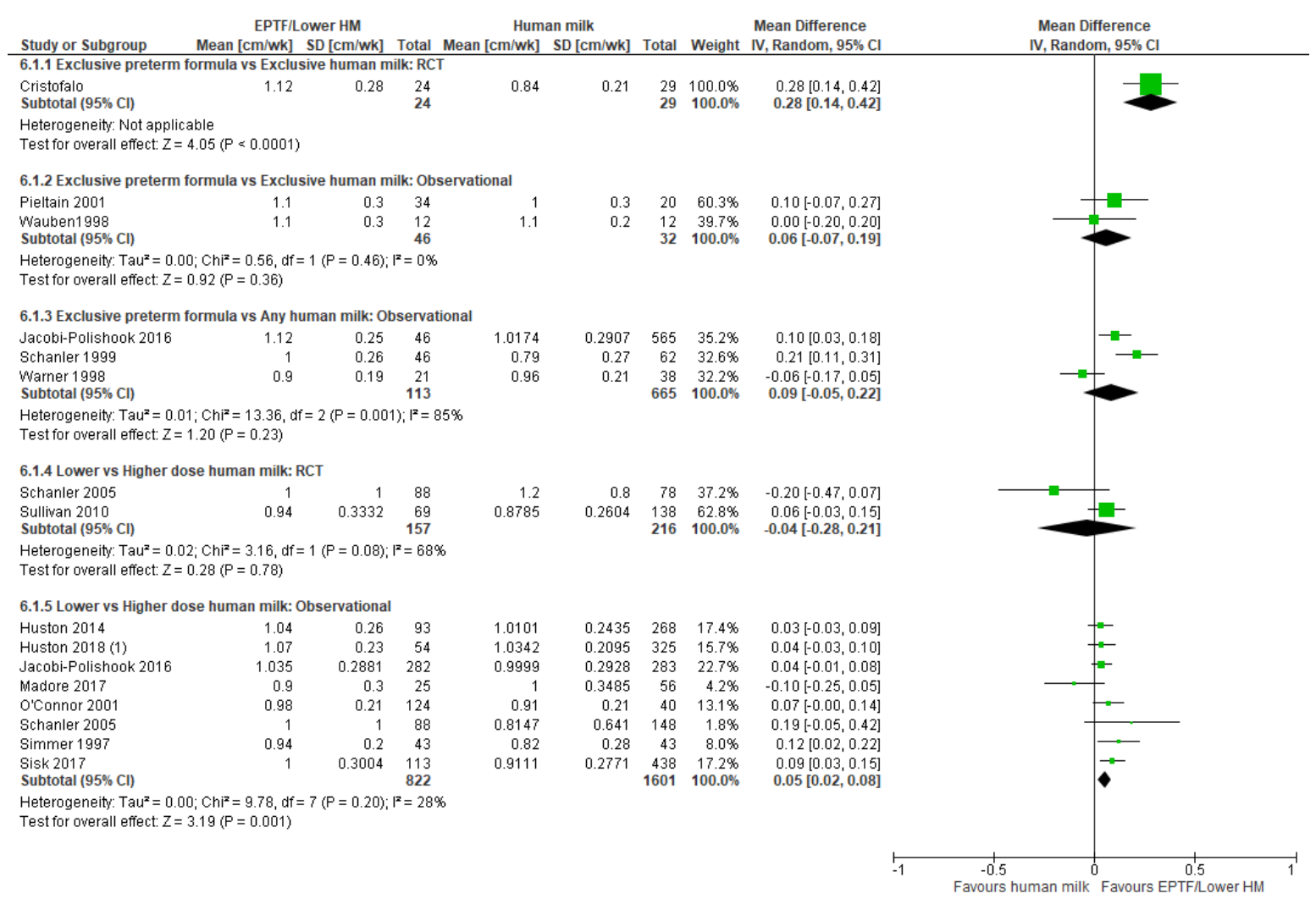

Footnotes

(1) Some overlap of infants with Huston 2014. When this study is excluded the result changes to $-0.04(-0.09,0.0)$

Figure 6. Forest plot of mean difference in change in length gain ( $\mathrm{cm} / \mathrm{wk})$ and human milk intake.

Observational studies: The impact of EPTF vs. EHM feeding on linear growth (cm/wk) was addressed in two studies [21,57]. On meta-analysis, there was no clear difference in linear growth between groups (MD 0.06, 95\% CI -0.07 to $0.19, n=78, \mathrm{I}^{2}=0 \%$, Figure 6, very low certainty evidence, Table S7).

Nicholl 1999 [35] investigated the effect of feeding variation on lower leg length gain from the time of enteral feeds reaching $\geq 150 \mathrm{~mL} / \mathrm{kg} / \mathrm{d}$ until nasogastric feeds ceased. There was no difference between groups (Table S1).

Change in length z-scores were reported in one study [55] with no clear difference detected (MD 0.00, 95\%CI -0.63 to $0.63, n=32$, Figure 7 , low risk of bias, Table S8).

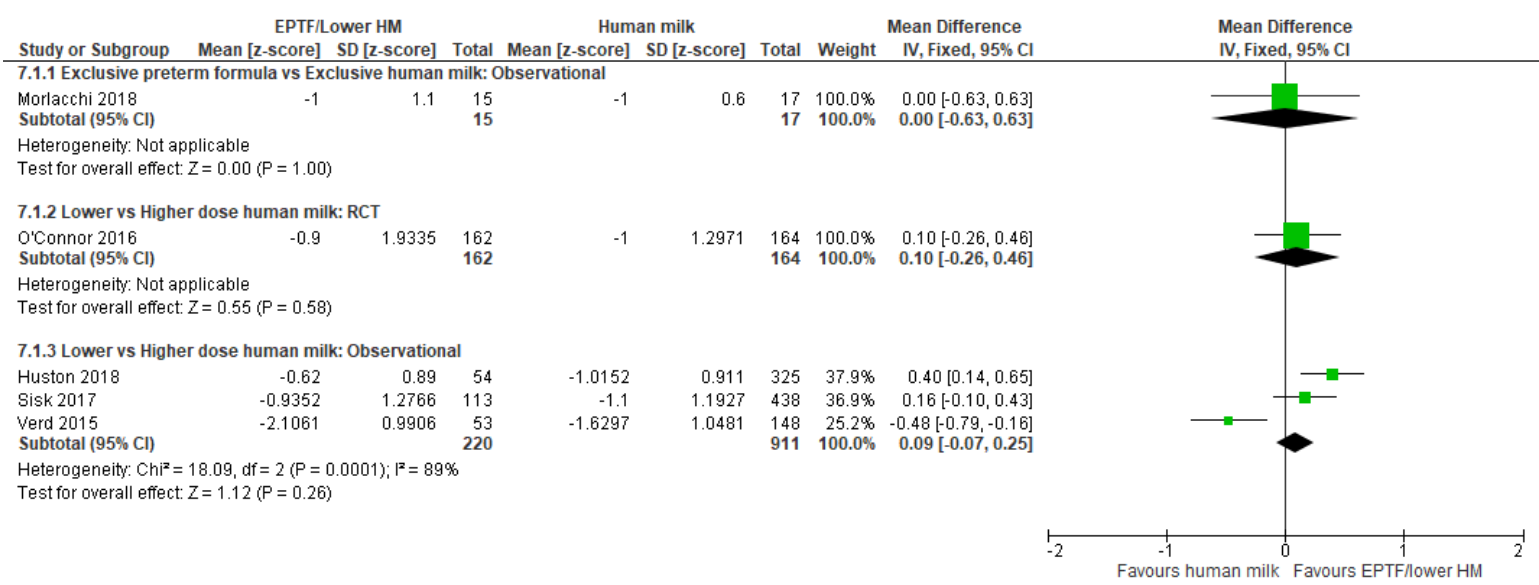

Figure 7. Forest plot of mean difference in change in length z-scores and human milk intake. 
Overall: The evidence for an effect of feeding EPTF vs. EHM on length gain is inconclusive.

\subsubsection{Comparison 2: Exclusive Preterm Formula vs. Any Human Milk}

Randomised trials: There were no RCTs identified reporting length gain for this comparison.

Observational studies: Three studies $[9,37,64]$ reported length gain $(\mathrm{cm} / \mathrm{wk})$ for this comparison and were included in the meta-analysis. No clear difference in length gain was shown between groups (MD 0.09, 95\% CI -0.05 to $0.22, n=778, \mathrm{I}^{2}=85 \%$, Figure 6, very low certainty evidence, Table S7). Heterogeneity may be explained by different dosages of HM.

Overall: The evidence for an effect of feeding EPTF vs. any HM on length gain is inconclusive.

\subsubsection{Comparison 3: Lower- vs. Higher-Dose Human Milk}

Randomised trials: No clear difference was shown in the meta-analysis of two RCTs [33,34] reporting length gain (cm/wk) (MD $-0.04,95 \% \mathrm{CI}-0.28$ to $0.21, n=373$, $\mathrm{I}^{2}=68 \%$, Figure 6 , low certainty evidence, Table S7). One RCT [32] reported change in length z-score and showed no clear difference between lower and higher-dose HM (MD $0.10,95 \%$ CI -0.26 to $0.46, n=326$, Figure 7 , low risk of bias, Table S8).

Observational studies: Nine studies reported length gain $(\mathrm{cm} / \mathrm{wk})$ with eight included in the meta-analysis $[20,28,29,33,37,52,59,61]$. Infants fed lower-dose HM compared with a higher dose showed a slightly higher length gain (MD $0.05,95 \%$ CI 0.02 to 0.08 , $n=2423, \mathrm{I}^{2}=24 \%$, Figure 6, low certainty evidence, Table S7).

Two studies [36,43] were unable to be included in the meta-analysis. Brownell 2018 [43] reported length velocity using MOM as reference, and neither the proportion of DHM nor PTF intake were associated with length gain (Table S1). Kaempf 1998 [36] reported the effect of fortified BM ( $>80 \%$ fortified BM) vs. PTF $(>80 \%$ PTF) feeding on lower leg gain $(\mathrm{mm} / \mathrm{d})$ and found no difference between groups (Table S1).

Eight studies reported length as change in z-scores, with three included in the metaanalysis $[28,42,61]$. There was no clear difference in change in length z-scores between groups (MD $0.09,95 \% \mathrm{CI}-0.07$ to $0.25, n=1131, \mathrm{I}^{2}=89 \%$, Figure 7 , very low certainty evidence, Table S8). For the remaining five studies, the results were variable, with two studies $[41,46]$ showing a greater increase in length z-scores with lower-dose HM and two studies showing no difference in change in length z-score [38,62], whereas Brownell 2018 reported that only PTF intake proportion was associated with decreased change in length $z$ scores (Table S1).

Overall: There is possibly no effect of lower vs. higher doses of HM feeding on length gain.

\subsection{Body Composition and Human Milk Feeding}

Body composition was reported in eight observational studies $[15,37,38,54,55,57,58,65]$ and measured using different techniques: two studies used dual-energy X-ray absorptiometry [57,65]; two used air displacement plethysmography [55,58]; two used either bioelectrical impedance analysis [15] or bioelectrical impedance spectroscopy [54]; one study used full body magnetic resonance imaging [38]; and one reported Body Mass Index (BMI) [37].

\subsubsection{Comparison 1: Exclusive Preterm Formula vs. Exclusive Human Milk}

Randomised trials: There were no RCTs identified for this comparison.

Observational studies: Three studies $[21,54,55]$ reported $\%$ fat-free mass and showed no clear difference between groups (MD $-1.46,95 \% \mathrm{CI}-4.35$ to $1.43, n=87, \mathrm{I}^{2}=80 \%$, Figure 8 , very low certainty evidence, Table S9). Four studies reported fat-free mass (g) $[15,54,55,57]$ and showed an increase with EPTF (MD 130.18, 95\% CI 53.86 to 206.50, $n=134, \mathrm{I}^{2}=0 \%$, Figure 9, very low certainty evidence, Table S10). 


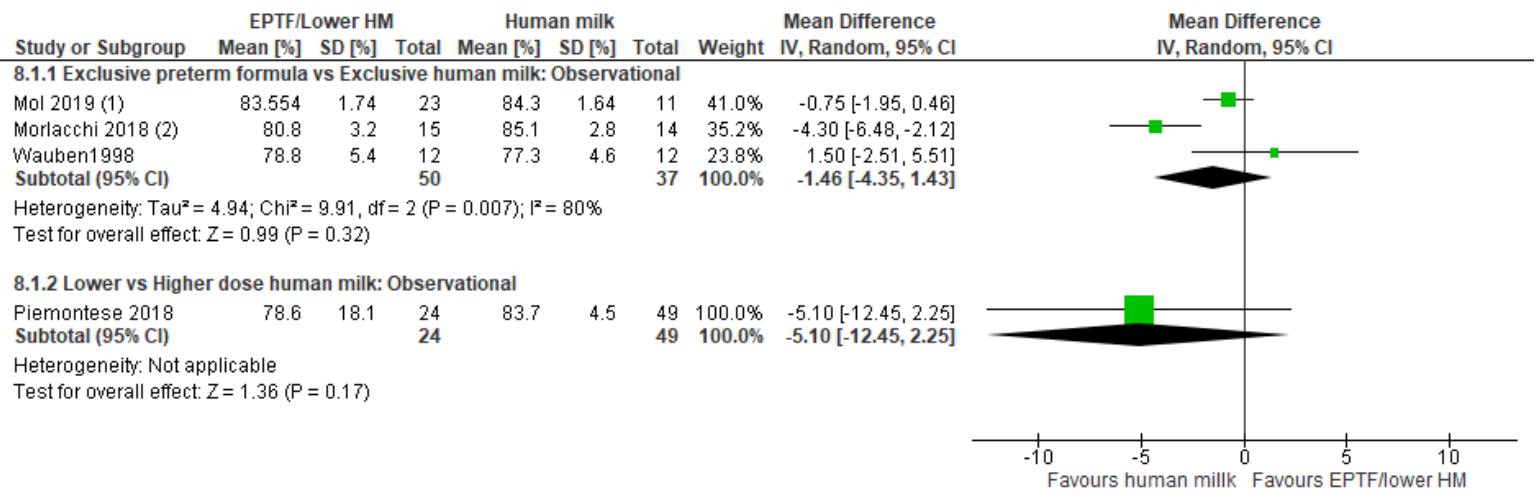

Footnotes

(1) Body composition measured at term corrected age by bioelectrical impedance spectroscopy

(2) Body composition measured at term corrected age by air displacement plethysmography

Figure 8. Forest plot of mean difference in change in $\%$ fat-free mass and human milk intake.

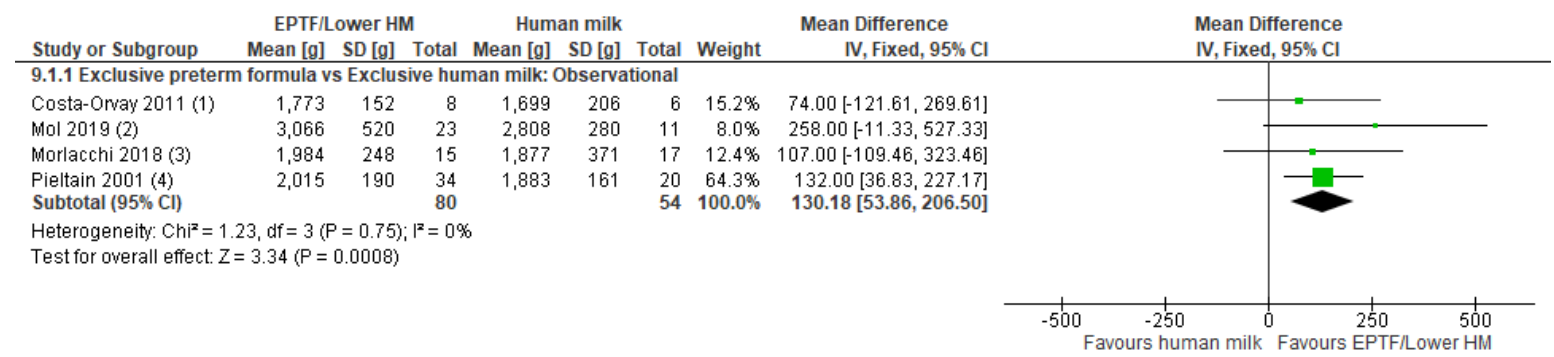

Footnotes

(1) At approx 36 weeks' gestation

(2) At term equivalent age

(3) At discharge

(4) At discharge

Figure 9. Forest plot of mean difference in change in fat-free mass $(\mathrm{g})$ and human milk intake.

Four studies $[54,55,57,65]$ reported $\%$ fat mass and showed no clear difference between groups (MD 1.82, 95\% CI -0.59 to $4.23, n=141, \mathrm{I}^{2}=83 \%$, Figure 10 , very low certainty evidence, Table S11). Four studies $[15,54,55,57]$ reported fat mass (g) and showed no clear difference between groups (MD 60.94, 95\% CI -5.42 to $127.31, n=134, \mathrm{I}^{2}=75 \%$, Figure 11 , very low certainty evidence, Table S12).

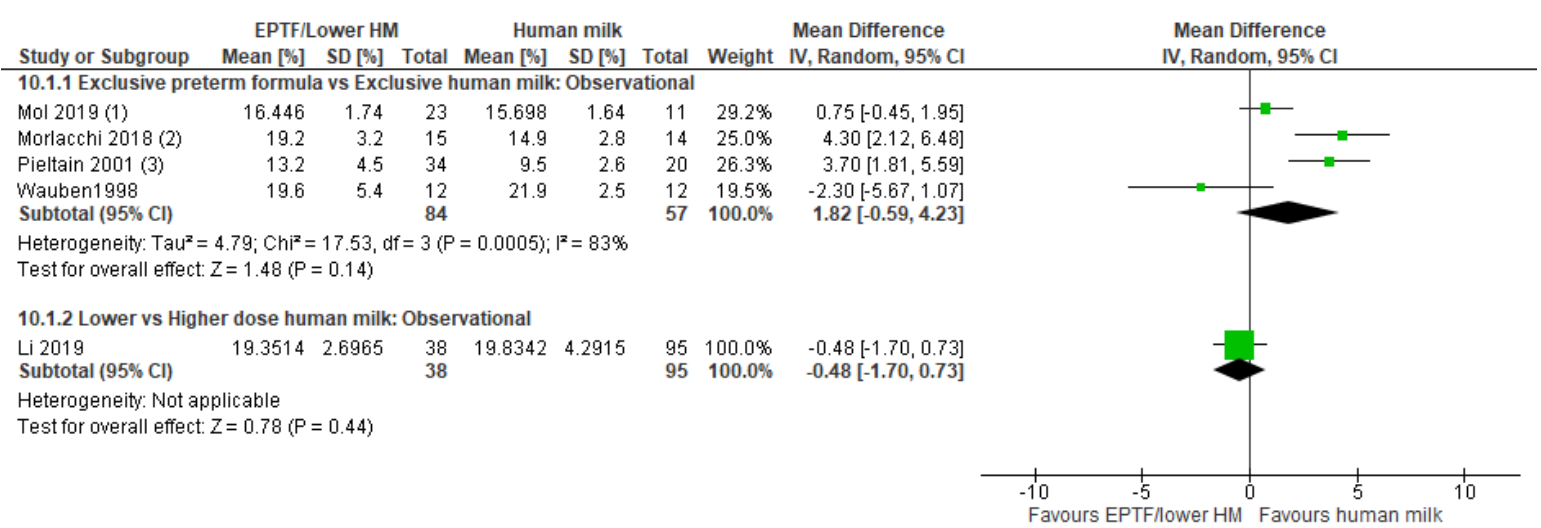

Footnotes

(1) Body composition measured at term equivalent age by bioelectrical impedance spectroscopy

(2) Body composition measured at term corrected age by air displacement plethysmography

(3) Body composition measured at discharge by dual energy $x$-ray absorptiometry

Figure 10. Forest plot of mean difference in change in $\%$ fat mass and human milk intake. 


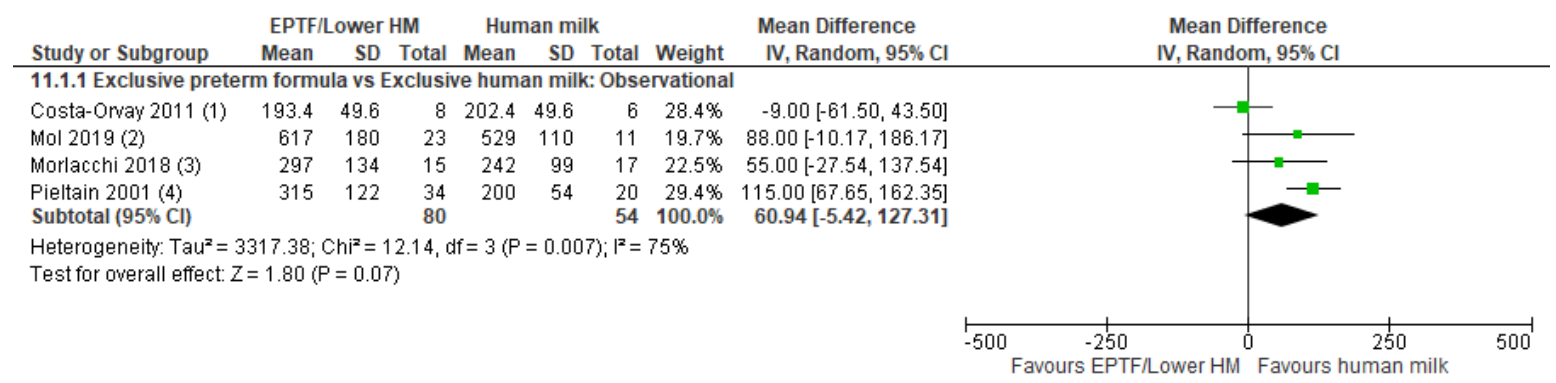

Footnotes

(1) At approx 36 weeks' gestation

(2) At term equivalent age

(3) At discharge

(4) At discharge

Figure 11. Forest plot of mean difference in change in fat mass (g) and human milk intake.

Overall: The evidence for an effect of feeding EPTF vs. EHM on fat and fat-free mass is inconclusive.

\subsubsection{Comparison 2: Any Human Milk vs. Exclusive Preterm Formula \\ There were no studies identified for this comparison.}

\subsubsection{Comparison 3: Lower- vs. Higher-Dose Human Milk Intake}

Piemontese 2018 [58] reported \% fat-free mass and showed no clear difference between groups (MD $-5.10,95 \% \mathrm{CI}-12.45$ to $2.25, n=73$, Figure 8 , low risk of bias, Table S9). Li 2019 [38] reported fat-free mass (g) with the predominantly formula-fed group having greater fat-free mass than EHM (MD 257.4, 95\% CI 139.1 to 357.7, $n=95, p<0.01$, Table S1).

Li 2019 [38] reported \% fat mass and showed no clear difference between groups (MD $-0.48,95 \% \mathrm{CI}-1.70$ to $0.73, n=133$, Figure 10, low risk of bias, Table S11). Li 2019 [38] also reported fat mass (g) with no clear difference between EHM and predominantly HM or predominantly formula (Table S1).

Jacobi-Polishook 2016 [37] reported BMI gain from birth to discharge $\left(\mathrm{kg} / \mathrm{m}^{2} / \mathrm{wk}\right)$ with similar BMI gain across quartile of HM intake (Table S1).

Overall: The evidence for an effect of feeding lower vs. higher-dose HM on fat and fat-free mass is inconclusive.

\section{Discussion}

\subsection{Summary of Main Results}

Forty-four studies were included in this review, of which 37 could be included in meta-analyses (4 RCTs with 866 infants and 33 observational studies with 9097 infants). Seven studies with 1917 infants were synthesised narratively. Overall, there was inconclusive evidence to draw reliable conclusions about the effect of HM feeding on growth outcomes in very low birth weight infants. There is a possible effect that lower doses of HM compared with higher doses of HM improve weight gain during the hospital admission; the overall quality of the evidence was low to very low for most outcomes; thus, our confidence in the results is limited. The majority of studies included in this review were categorised in the lower versus higher-dose HM comparison, with insufficient evidence to reliably assess the effect an exclusive HM diet versus EPTF on any outcomes.

We included measures of body composition in our review to examine the possible effects of HM feeding on quality of growth. Few studies reported these outcomes. The available evidence was poor quality but did suggest that the proportion of fat mass (\%) at term corrected age was significantly lower in EHM-fed vs. EPTF-fed infants, a positive effect of $\mathrm{HM}$, as lower fat mass at term corrected age better aligns with infants born at term [66]. These findings warrant confirmation in further large-scale studies and reiterate the need for inclusion of measures of not only growth velocity but also quality (e.g., fat-free mass) in studies examining long-term outcomes of preterm infants. 


\subsection{Findings from Other Reviews}

While there are a large number of studies reporting on growth among preterm infants fed with fortified HM, there are few systematic reviews published in this area. The available studies are largely observational; a recent Cochrane review examining the effects of formula vs. maternal milk feeding for preterm infants failed to identify any RCTs that met their criteria [10]. Another Cochrane review by Quigley and colleagues compared formula and donor breast milk for feeding preterm infants [11] and included a subgroup analysis of fortified donor HM with preterm formula, which is the closest match to our review. They also found an effect of higher growth rates in favour of preterm formula for all measures. The three studies that their review comprises [31-33] are also included in our meta-analysis.

Our review provides a more complete summary of the evidence concerning HM intake and growth outcomes as we included non-randomised study designs and did not apply any restrictions regarding the source of milk (e.g., MOM or DHM). Nevertheless, our findings regarding weight gain were similar to the effect size reported by Quigley et al. [11] (MD $2.37,95 \%$ CI 1.09 to 3.65$) \mathrm{g} / \mathrm{kg} / \mathrm{d}$ ), suggesting slower weight gain with cumulative HM intake. However, we rated the overall evidence as inconclusive, as many of the included studies were small and thus underpowered and/or had major methodological limitations.

Unlike Quigley et al. [11], we did not find consistent results for length gain, either when reported as cm/week or change in z-score; however, this was sparsely reported across different comparison groups, and thus should be interpreted with caution. We did identify a possible effect of increased HC gain with EPTF vs. any HM feeds, based on data predominantly from observational studies, which is also inconsistent with the findings of the Quigley review. However, findings were not consistent across HM exposure groups. For the comparison of low versus higher-dose HM, we concluded that there was possibly no effect of dose of HM on HC gain, based on moderate certainty of evidence generated from RCTs and trivial differences identified in the observational studies reporting this outcome. Notably, across the comparisons where head growth was reported, the pooled effect size was less than $0.1 \mathrm{~cm} /$ week. When calculated over a 12-week admission, this equates to a difference of just under $1 \mathrm{~cm}$ in head circumference: roughly equivalent to a centile space on intrauterine growth curves.

Previous studies have shown that faster head growth before term and post-discharge is associated with small improvements in longer-term neurodevelopmental outcomes [16,67]. This has led to an increased focus on early intensive parenteral and enteral nutritional support in preterm infants. However, Rozé and colleagues analysed data from two recent large cohort studies of nearly 3000 very preterm infants and identified inconsistencies in the relationship between early growth and developmental outcomes in breastfed infants, in what the authors term the 'apparent breastfeeding paradox' [68]. They found breastfeeding at discharge was associated with a 1.5-2.5 increase in the odds of losing one weight z-score during hospitalisation but with an increased odds of having an $\mathrm{HC}$ z-score higher than 0.5 at 2 years and a decreased risk of suboptimal neurodevelopment at 2 and 5 years of age, respectively [68]. Although not an outcome of this review, a previous meta-analysis found there is inconclusive evidence regarding a possible effect of HM feedings on cognitive and motor development in VLBW infants [3].

\subsection{Implications for Clinical Practice and Research}

HM should continue to be promoted as the optimal source of nutrition for all infants, given the known benefits beyond infant growth. However, further high-quality research is needed to delineate the complex relationships between infant feeding practices, weight gain, body composition, and later neurodevelopment in VLBW infants. In particular, clarity is needed regarding the optimal ways to feed expressed HM to VLBW infants in the early weeks and months after birth. The inconclusive findings identified in this review are likely heavily influenced by differences in feeding management between studies, given the variety of settings in which the studies were conducted. This includes practices regarding 
fortification of expressed breast milk, including individual versus standardised fortification regimens, as well as changes in the makeup of commercially available fortifiers.

The protein concentration of fortifiers has generally increased over time, and there is evidence of small increases in weight in hospital with the use of higher protein versus lower protein concentration fortifiers [69]. While we selected studies that used contemporary feeding approaches such as fortified HM, the level of fortification varied considerably and was often not reported. The source of the fortifier also varied, with some recent studies using a human milk-derived fortifier rather than the more commonly available commercial bovine milk-derived fortifiers. Recent meta-analyses indicate potential clinical benefits associated with the use of a human milk-derived vs. bovine milk-derived fortifier, although only one study reported on growth outcomes and found no difference [70,71].

In addition, there was significant variation in practices surrounding the use of DHM between studies. This has the potential to influence growth outcomes, as the protein concentration of DHM is highly variable and influenced by the lactational stage of the donors [72]. The pasteurisation and storage practices concerning DHM are also known to affect the concentration of bioactive proteins and other components of HM [73].

Future studies should be sufficiently large enough to examine the effects of an exclusive HM diet, as well as potential threshold and dose-response relationships, on growth and longer-term developmental outcomes in preterm infants. Studies must include detailed description of feeding management practices to permit a more accurate estimate of protein and energy intakes among participants. This will generate evidence to better define the cumulative effects of HM feeds that will help inform the optimal feeding strategies in the neonatal unit, particularly while breastfeeding is being established. In addition, detailed collection and reporting based on the source of HM (mother's own or donor) are needed to clarify any specific impact of DHM feeds on growth and neurodevelopmental outcomes.

\subsection{Strengths and Limitations}

We have used robust methods to systematically search, synthesise, and critique the evidence. At least two reviewers independently abstracted data and assessed the quality and certainty of evidence using established tools. However, it is nevertheless possible that studies were not identified.

There are several limitations. There is considerable heterogeneity, both clinical and statistical, in the included studies. This in part reflects our deliberate approach to allow a range of $\mathrm{HM}$ exposures and a broad range of outcome measures. For example, there was some variation in the volume of HM intake among studies included in the 'any HM vs. PTF' and 'high- vs. low-dose' comparisons, and in some cases, the volume was not specifically reported. In addition, growth was measured over varying time points, and different protocols for length, head circumference, and body composition measurement between studies may have led to measurement errors and thus heterogeneity in the meta-analyses.

Differences in clinical management, including feeding practices, as described earlier, are another significant source of heterogeneity. Teasing out the effects of fortification and DHM was not possible in this review, as often these practices were poorly described in individual studies.

Most of the evidence reviewed is from observational studies, including five RCTs where comparisons relevant to the review included a non-randomised arm and therefore carry an increased risk of bias. We deliberately included both RCTs and observational studies in this review to provide a comprehensive synthesis of the available evidence, and we have accounted for study design by using the GRADE system to decide the certainty of evidence. Nevertheless, many of the studies had small sample sizes and included growth as secondary outcomes. Interpretation of the evidence is complex, particularly in comparisons with data from both RCTs and observational studies, where the effect is not in alignment. For this reason, we took a conservative approach and defined our interpretation of the evidence a priori to ensure consistency. 


\section{Conclusions}

Although we identified a large number of studies involving over 10,000 VLBW infants, we have found much of the evidence for the association between growth outcomes and HM intake to be inconclusive, largely due to the quality of the evidence. While the metaanalysis demonstrated possible effects of increased weight gain among infants fed lower doses of HM and increased head circumference gain among those fed EPTF vs. any HM, the certainty of the body of the available evidence was very low to low. The effect sizes were also small; thus, the clinical significance of these differences is unclear. Carefully designed studies that assess dose-dependent effects and account for the source of milk and specific protein and energy fortification practices are needed to inform optimal HM feeding strategies in the neonatal unit.

Supplementary Materials: The following are available online at https:/ /www.mdpi.com/article/10 .3390/nu13062089/s1, Figure S1: Prisma diagram—selection of studies, Table S1: Results of studies synthesised narratively, Table S2: Summary of findings: Preterm formula vs. Human milk—Weight gain (g/d), Table S3: Summary of findings: Preterm formula vs. Human milk-Weight gain (g/kg/d), Table S4: Summary of findings: Preterm formula vs. Human milk-Change in weight z-score, Table S5: Summary of findings: Preterm formula vs. Human milk title-Head circumference gain, Table S6: Summary of findings: Preterm formula vs. Human milk-Change in head circumference z-score, Table S7: Summary of findings: Preterm formula vs. Human milk-Length gain (cm/week), Table S8: Summary of findings: Preterm formula vs. Human milk-Change in length z-score, Table S9: Summary of findings: Preterm formula vs. Human milk-\% Fat-free mass, Table S10: Summary of findings: Preterm formula vs. Human milk-Fat-free mass (g), Table S11: Summary of findings: Preterm formula vs. Human milk—\% fat mass, Table S12: Summary of findings: Preterm formula vs. Human milk-Fat mass (g).

Author Contributions: Conceptualisation, M.S., J.M., and C.T.C.; methodology, J.M., A.R.R., and C.T.C.; quality assessment: M.S., J.M., and C.T.C.; data extraction and meta-analysis: M.S., Y.F.C., J.M., A.R.R., and C.T.C.; writing — original draft preparation, M.S. and J.M.; writing—review and editing, all authors. All authors have read and agreed to the published version of the manuscript.

Funding: This research received no external funding.

Conflicts of Interest: C.C. salary was supported by a Research Fellowship (ID 1132596) from the National Health and Medical Research Council (NHMRC), Australia. The views expressed in this article are solely the responsibility of the authors and do not reflect the views of the NHMRC. C.C. is an author on an included paper (Jacobi-Polishook 2016). All other authors declare no conflict of interest.

\section{References}

1. Edmond, K.; Bahl, R. Optimal Feeding of Low-Birth-Weight Infants; Technical Review; World Health Organisation: Geneva, Switzerland, 2006.

2. Agostoni, C.; Buonocore, G.; Carnielli, V.P.; de Curtis, M.; Darmaun, D.; Decsi, T.; Domellof, M.; Embleton, N.D.; Fusch, C.; Genzel-Boroviczeny, O.; et al. Enteral nutrient supply for preterm infants: Commentary from the european society of paediatric gastroenterology, hepatology and nutrition committee on nutrition. J. Pediatr. Gastroenterol. Nutr. 2010, 50, 85-91. [CrossRef] [PubMed]

3. Miller, J.; Tonkin, E.; Damarell, R.; McPhee, A.; Suganuma, M.; Suganuma, H.; Middleton, P.; Makrides, M.; Collins, C. A systematic review and meta-analysis of human milk feeding and morbidity in very low birth weight infants. Nutrients 2018, 10, 707. [CrossRef] [PubMed]

4. AAP Committee on Nutrition; AAP Section on Breastfeeding; AAP Committee on Fetus and Newborn. Donor human milk for the high-risk infant: Preparation, safety, and usage options in the United States. Pediatrics 2017, 139, e20163440. [CrossRef] [PubMed]

5. Brown, J.V.; Lin, L.; Embleton, N.D.; Harding, J.E.; McGuire, W. Multi-nutrient fortification of human milk for preterm infants. Cochrane Database Syst. Rev. 2020, 5, CD000343. [CrossRef]

6. Arslanoglu, S.; Boquien, C.-Y.; King, C.; Lamireau, D.; Tonetto, P.; Barnett, D.; Bertino, E.; Gaya, A.; Gebauer, C.; Grovslien, A.; et al. Fortification of human milk for preterm infants: Update and recommendations of the european milk bank association (emba) working group on human milk fortification. Front. Pediatr. 2019, 7, 76. [CrossRef] [PubMed]

7. Colaizy, T.T.; Carlson, S.; Saftlas, A.F.; Morriss, F.H., Jr. Growth in vlbw infants fed predominantly fortified maternal and donor human milk diets: A retrospective cohort study. BMC Pediatr. 2012, 12, 124. [CrossRef] 
8. Ginovart, G.; Gich, I.; Gutierrez, A.; Verd, S. A fortified donor milk policy is associated with improved in-hospital head growth and weight gain in very low-birth-weight infants. Adv. Neonatal Care 2017, 17, 250-257. [CrossRef]

9. Schanler, R.J.; Shulman, R.J.; Lau, C. Feeding strategies for premature infants: Beneficial outcomes of feeding fortified human milk versus preterm formula. Pediatrics 1999, 103, 1150-1157. [CrossRef]

10. Brown, J.V.E.; Walsh, V.; McGuire, W. Formula versus maternal breast milk for feeding preterm or low birth weight infants. Cochrane Database Syst. Rev. 2019, 8, CD002972. [CrossRef]

11. Quigley, M.; Embleton, N.D.; McGuire, W. Formula versus donor breast milk for feeding preterm or low birth weight infants. Cochrane Database Syst. Rev. 2019, 7, CD002971. [CrossRef]

12. Huang, P.; Zhou, J.; Yin, Y.; Jing, W.; Luo, B.; Wang, J. Effects of breast-feeding compared with formula-feeding on preterm infant body composition: A systematic review and meta-analysis. Br. J. Nutr. 2016, 116, 132-141. [CrossRef]

13. Leppanen, M.; Lapinleimu, H.; Lind, A.; Matomaki, J.; Lehtonen, L.; Haataja, L.; Rautava, P.; Group, P.S. Antenatal and postnatal growth and 5-year cognitive outcome in very preterm infants. Pediatrics 2014, 133, 63-70. [CrossRef] [PubMed]

14. Moher, D.; Liberati, A.; Tetzlaff, J.; Altman, D.G.; Group, P. Preferred reporting items for systematic reviews and meta-analyses: The prisma statement. BMJ 2009, 339, b2535. [CrossRef]

15. Costa-Orvay, J.A.; Figueras-Aloy, J.; Romera, G.; Closa-Monasterolo, R.; Carbonell-Estrany, X. The effects of varying protein and energy intakes on the growth and body composition of very low birth weight infants. Nutr. J. 2011, 10, 140. [CrossRef] [PubMed]

16. Belfort, M.B.; Rifas-Shiman, S.L.; Sullivan, T.; Collins, C.T.; McPhee, A.J.; Ryan, P.; Kleinman, K.P.; Gillman, M.W.; Gibson, R.A.; Makrides, M. Infant growth before and after term: Effects on neurodevelopment in preterm infants. Pediatrics 2011, 128, e899-e906. [CrossRef]

17. Cormack, B.E.; Embleton, N.D.; van Goudoever, J.B.; Hay, W.W., Jr.; Bloomfield, F.H. Comparing apples with apples: It is time for standardized reporting of neonatal nutrition and growth studies. Pediatr. Res. 2016, 79, 810-820. [CrossRef]

18. Covidence Systematic Review Software; Veritas Health Innovation: Melbourne, Australia. Available online: www.covidence.org (accessed on 20 June 2020).

19. Fewtrell, M.S.; Morley, R.; Abbott, R.A.; Singhal, A.; Isaacs, E.B.; Stephenson, T.; MacFadyen, U.; Lucas, A. Double-blind, randomized trial of long-chain polyunsaturated fatty acid supplementation in formula fed to preterm infants. Pediatrics 2002, 110, 73-82. [CrossRef]

20. O'Connor, D.L.; Hall, R.T.; Adamkin, D.H.; Auestad, N.; Castillo, M.; Connor, W.E.; Connor, S.L.; Fitzgerald, K.M.; Groh-Wargo, S.; Hartmann, E.E.; et al. Growth and development in preterm infants fed long-chain polyunsaturated fatty acids: A prospective, randomized controlled trial. Pediatrics 2001, 108, 359-371. [CrossRef]

21. Wauben, I.P.; Atkinson, S.A.; Grad, T.L.; Shah, J.K.; Paes, B. Moderate nutrient supplementation of mother's milk for preterm infants supports adequate bone mass and short-term growth: A randomized, controlled trial. Am. J. Clin. Nutr. 1998, 67, 465-472. [CrossRef] [PubMed]

22. Lok, K.Y.W.; Chau, P.H.; Fan, H.S.L.; Chan, K.M.; Chan, B.H.; Fung, G.P.C.; Tarrant, M. Increase in weight in low birth weight and very low birth weight infants fed fortified breast milk versus formula milk: A retrospective cohort study. Nutrients 2017, 9, 520. [CrossRef]

23. Higgins, J.P.T.; Thomas, J.; Chandler, J.; Cumpston, M.; Li, T.; Page, M.J.; Welch, V.A. (Eds.) Cochrane Handbook for Systematic Reviews of Interventions; John Wiley and Sons: Hoboken, NI, USA. Available online: www.training.cochrane.org/handbook (accessed on 20 April 2020).

24. Academy of Nutrition and Dietetics. Evidence Analysis Manual: Steps in the Academy Evidence Analysis Process; Academy of Nutrition and Dietetics: Chicago, IL, USA, 2016.

25. Review Manager (RevMan) [Computer Program]; Version 5.4; The Cochrane Collaboration: Copenhagen, Denmark; The Nordic Cochrane Centre: Copenhagen, Denmark, 2020.

26. Luo, D.; Wan, X.; Liu, J.; Tong, T. Optimally estimating the sample mean from the sample size, median, mid-range, and/or mid-quartile range. Stat. Methods Med. Res. 2016, 27, 1785-1805. [CrossRef]

27. Wan, X.; Wang, W.; Liu, J.; Tong, T. Estimating the sample mean and standard deviation from the sample size, median, range and/or interquartile range. BMC Med. Res. Methodol. 2014, 14, 135. [CrossRef]

28. Huston, R.K.; Markell, A.M.; McCulley, E.A.; Gardiner, S.K.; Sweeney, S.L. Improving growth for infants $\leq 250$ grams receiving an exclusive human milk diet. Nutr. Clin. Pract. 2018, 33, 671-678. [CrossRef]

29. Huston, R.K.; Markell, A.M.; McCulley, E.A.; Pathak, M.; Rogers, S.P.; Sweeney, S.L.; Dolphin, N.G.; Gardiner, S.K. Decreasing necrotizing enterocolitis and gastrointestinal bleeding in the neonatal intensive care unit: The role of donor human milk and exclusive human milk diets in infants $\leq 1500 \mathrm{~g}$ birth weight. Infant Child. Adolesc. Nutr. 2014, 6, 86-93. [CrossRef]

30. Gradepro Gdt: Gradepro Guideline Development Tool [Software]; McMaster University: Hamilton, ON, Canada, 2015; (developed by Evidence Prime, Inc.); Available online: gradepro.org (accessed on 23 November 2020).

31. Cristofalo, E.A.; Schanler, R.J.; Blanco, C.L.; Sullivan, S.; Trawoeger, R.; Kiechl-Kohlendorfer, U.; Dudell, G.; Rechtman, D.J.; Lee, M.L.; Lucas, A.; et al. Randomized trial of exclusive human milk versus preterm formula diets in extremely premature infants. J. Pediatr. 2013, 163, 1592-1595.e1591. [CrossRef]

32. O'Connor, D.L.; Gibbins, S.; Kiss, A.; Bando, N.; Brennan-Donnan, J.; Ng, E.; Campbell, D.M.; Vaz, S.; Fusch, C.; Asztalos, E.; et al. Effect of supplemental donor human milk compared with preterm formula on neurodevelopment of very low-birth-weight infants at 18 months: A randomized clinical trial. JAMA 2016, 316, 1897-1905. [CrossRef] 
33. Schanler, R.J.; Lau, C.; Hurst, N.M. Randomized trial of donor human milk versus preterm formula as substitutes for mothers' own milk in the feeding of extremely premature infants. Pediatrics 2005, 116, 400-406. [CrossRef] [PubMed]

34. Sullivan, S.; Schanler, R.J.; Kim, J.H.; Patel, A.L.; Trawöger, R.; Kiechl-Kohlendorfer, U.; Chan, G.M.; Blanco, C.L.; Abrams, S.; Cotten, C.M.; et al. An exclusively human milk-based diet is associated with a lower rate of necrotizing enterocolitis than a diet of human milk and bovine milk-based products. J. Pediatr. 2010, 156, 562-567.e561. [CrossRef] [PubMed]

35. Nicholl, R.M.; Gamsu, H.R. Changes in growth and metabolism in very low birthweight infants fed with fortified breast milk. Acta Paediatr. 1999, 88, 1056-1061. [CrossRef] [PubMed]

36. Kaempf, D.E.; Pfluger, M.S.; Thiele, A.M.; Hermanussen, M.; Linderkamp, O. Influence of nutrition on growth in premature infants: Assessment by knemometry. Ann. Hum. Biol. 1998, 25, 127-136. [CrossRef]

37. Jacobi-Polishook, T.; Collins, C.T.; Sullivan, T.R.; Simmer, K.; Gillman, M.W.; Gibson, R.A.; Makrides, M.; Belfort, M.B. Human milk intake in preterm infants and neurodevelopment at 18 months corrected age. Pediatr. Res. 2016, 80, 486-492. [CrossRef] [PubMed]

38. Li, Y.; Liu, X.; Modi, N.; Uthaya, S. Impact of breast milk intake on body composition at term in very preterm babies: Secondary analysis of the nutritional evaluation and optimisation in neonates randomised controlled trial. Arch. Dis. Child. Fetal. Neonatal Ed. 2019, 104, F306-F312. [CrossRef] [PubMed]

39. Assad, M.; Elliott, M.J.; Abraham, J.H. Decreased cost and improved feeding tolerance in vlbw infants fed an exclusive human milk diet. J. Perinatol. 2016, 36, 216-220. [CrossRef] [PubMed]

40. Colacci, M.; Murthy, K.; DeRegnier, R.O.; Khan, J.Y.; Robinson, D.T. Growth and development in extremely low birth weight infants after the introduction of exclusive human milk feedings. Am. J. Perinatol. 2017, 34, 130-137. [CrossRef]

41. Hoban, R.; Schoeny, M.E.; Esquerra-Zwiers, A.; Kaenkumchorn, T.K.; Casini, G.; Tobin, G.; Siegel, A.H.; Patra, K.; Hamilton, M.; Wicks, J.; et al. Impact of donor milk on short- and long-term growth of very low birth weight infants. Nutrients 2019, 11, 241. [CrossRef]

42. Verd, S.; Porta, R.; Botet, F.; Gutierrez, A.; Ginovart, G.; Barbero, A.H.; Ciurana, A.; Plata, II. Hospital outcomes of extremely low birth weight infants after introduction of donor milk to supplement mother's milk. Breastfeed. Med. 2015, 10, 150-155. [CrossRef]

43. Brownell, E.A.; Matson, A.P.; Smith, K.C.; Moore, J.E.; Esposito, P.A.; Lussier, M.M.; Lerer, T.J.; Hagadorn, J.I. Doseresponse relationship between donor human milk, mother's own milk, preterm formula, and neonatal growth outcomes. J. Pediatr. Gastroenterol. Nutr. 2018, 67, 90-96. [CrossRef]

44. Cañizo Vázquez, D.; Salas García, S.; Izquierdo Renau, M.; Iglesias-Platas, I. Availability of donor milk for very preterm infants decreased the risk of necrotizing enterocolitis without adversely impacting growth or rates of breastfeeding. Nutrients 2019, 11, 1895. [CrossRef]

45. Carlson, S.J.; Ziegler, E.E. Nutrient intakes and growth of very low birth weight infants. J. Perinatol. 1998, 18, $252-258$.

46. Castellano Yáñez, C.; Castillo Barrio, B.; Muñoz Labián, M.D.C.; Ortiz Movilla, R.; García Lara, N.R.; Royuela Vicente, A.; Marín Gabriel, M.A. Providing very preterm infants with donor human milk led to faster breastfeeding rates but worse biometric gains. Acta Paediatr. 2019, 108, 766-767. [CrossRef]

47. Chowning, R.; Radmacher, P.; Lewis, S.; Serke, L.; Pettit, N.; Adamkin, D.H. A retrospective analysis of the effect of human milk on prevention of necrotizing enterocolitis and postnatal growth. J. Perinatol. 2016, 36, 221-224. [CrossRef] [PubMed]

48. Hair, A.B.; Peluso, A.M.; Hawthorne, K.M.; Perez, J.; Smith, D.P.; Khan, J.Y.; O’Donnell, A.; Powers, R.J.; Lee, M.L.; Abrams, S.A. Beyond necrotizing enterocolitis prevention: Improving outcomes with an exclusive human milk-based diet. Breastfeed. Med. 2016, 11, 70-74. [CrossRef]

49. Lee, L.Y.; Lee, J.; Niduvaje, K.; Seah, S.S.; Atmawidjaja, R.W.; Cheah, F.C. Nutritional therapies in the neonatal intensive care unit and post-natal growth outcomes of preterm very low birthweight asian infants. J. Paediatr. Child. Health 2020, 56, 400-407. [CrossRef] [PubMed]

50. Levene, I.; McCormick, K. Improved growth of extremely and very preterm babies: Evaluation of a quality-of-care initiative. J. Paediatr. Child. Health 2020, 56, 444-449. [CrossRef] [PubMed]

51. Maas, C.; Wiechers, C.; Bernhard, W.; Poets, C.F.; Franz, A.R. Early feeding of fortified breast milk and in-hospital-growth in very premature infants: A retrospective cohort analysis. BMC Pediatr. 2013, 13, 178. [CrossRef]

52. Madore, L.S.; Bora, S.; Erdei, C.; Jumani, T.; Dengos, A.R.; Sen, S. Effects of donor breastmilk feeding on growth and early neurodevelopmental outcomes in preterm infants: An observational study. Clin. Ther. 2017, 39, 1210-1220. [CrossRef]

53. Manea, A.; Boia, M.; Iacob, D.; Dima, M.; Iacob, R.E. Benefits of early enteral nutrition in extremely low birth weight infants. Singapore Med. J. 2016, 57, 616-618. [CrossRef]

54. Mol, N.; Zasada, M.; Kwinta, P. Does type of feeding affect body composition in very low birth weight infants?-A prospective cohort study. Pediatr. Neonatol. 2019, 60, 135-140. [CrossRef]

55. Morlacchi, L.; Roggero, P.; Gianni, M.L.; Bracco, B.; Porri, D.; Battiato, E.; Menis, C.; Liotto, N.; Mallardi, D.; Mosca, F. Protein use and weight-gain quality in very-low-birth-weight preterm infants fed human milk or formula. Am. J. Clin. Nutr. 2018, 107, 195-200. [CrossRef]

56. Petrova, A.; Eccles, S.; Mehta, R. Role of the proportional intake of fortified mother's own milk in the weight gain pattern of their very-preterm-born infants. Nutrients 2020, 12, 1571. [CrossRef]

57. Pieltain, C.; de Curtis, M.; Gerard, P.; Rigo, J. Weight gain composition in preterm infants with dual energy x-ray absorptiometry. Pediatr. Res. 2001, 49, 120-124. [CrossRef] [PubMed] 
58. Piemontese, P.; Liotto, N.; Mallardi, D.; Roggero, P.; Puricelli, V.; Gianni, M.L.; Morniroli, D.; Tabasso, C.; Perrone, M.; Menis, C.; et al. The effect of human milk on modulating the quality of growth in preterm infants. Front. Pediatr. 2018, 6, 291. [CrossRef]

59. Simmer, K.; Metcalf, R.; Daniels, L. The use of breastmilk in a neonatal unit and its relationship to protein and energy intake and growth. J. Paediatr. Child. Health 1997, 33, 55-60. [CrossRef] [PubMed]

60. Sisk, P.M.; Lovelady, C.A.; Gruber, K.J.; Dillard, R.G.; O'Shea, T.M. Human milk consumption and full enteral feeding among infants who weigh $\leq 1250$ grams. Pediatrics 2008, 121, e1528-e1533. [CrossRef] [PubMed]

61. Sisk, P.M.; Lambeth, T.M.; Rojas, M.A.; Lightbourne, T.; Barahona, M.; Anthony, E.; Auringer, S.T. Necrotizing enterocolitis and growth in preterm infants fed predominantly maternal milk, pasteurized donor milk, or preterm formula: A retrospective study. Am. J. Perinatol. 2017, 34, 676-683.

62. Soldateli, B.; Parker, M.; Melvin, P.; Gupta, M.; Belfort, M. Human milk feeding and physical growth in very low-birth-weight infants: A multicenter study. J. Perinatol. 2020, 40, 1246-1252. [CrossRef]

63. Spiegler, J.; Preuss, M.; Gebauer, C.; Bendiks, M.; Herting, E.; Gopel, W.; German Neonatal Network. Does breastmilk influence the development of bronchopulmonary dysplasia? J. Pediatr. 2016, 169, 76-80.e74. [CrossRef]

64. Warner, J.T.; Linton, H.R.; Dunstan, F.D.; Cartlidge, P.H. Growth and metabolic responses in preterm infants fed fortified human milk or a preterm formula. Int. J. Clin. Pract. 1998, 52, 236-240.

65. Wauben, I.P.; Atkinson, S.A.; Shah, J.K.; Paes, B. Growth and body composition of preterm infants: Influence of nutrient fortification of mother's milk in hospital and breastfeeding post-hospital discharge. Acta Paediatr. 1998, 87, 780-785. [CrossRef]

66. Hamatschek, C.; Yousuf, E.I.; Mollers, L.S.; So, H.Y.; Morrison, K.M.; Fusch, C.; Rochow, N. Fat and fat-free mass of preterm and term infants from birth to six months: A review of current evidence. Nutrients 2020, 12, 288. [CrossRef]

67. Franz, A.R.; Pohlandt, F.; Bode, H.; Mihatsch, W.A.; Sander, S.; Kron, M.; Steinmacher, J. Intrauterine, early neonatal, and postdischarge growth and neurodevelopmental outcome at 5.4 years in extremely preterm infants after intensive neonatal nutritional support. Pediatrics 2009, 123, e101-e109. [CrossRef] [PubMed]

68. Roze, J.C.; Darmaun, D.; Boquien, C.Y.; Flamant, C.; Picaud, J.C.; Savagner, C.; Claris, O.; Lapillonne, A.; Mitanchez, D.; Branger, B.; et al. The apparent breastfeeding paradox in very preterm infants: Relationship between breast feeding, early weight gain and neurodevelopment based on results from two cohorts, epipage and lift. BMJ Open 2012, 2, e000834. [CrossRef] [PubMed]

69. Gao, C.; Miller, J.; Collins, C.T.; Rumbold, A.R. Comparison of different protein concentrations of human milk fortifier for promoting growth and neurological development in preterm infants. Cochrane Database Syst. Rev. 2020, 11, CD007090. [CrossRef]

70. Premkumar, M.H.; Pammi, M.; Suresh, G. Human milk-derived fortifier versus bovine milk-derived fortifier for prevention of mortality and morbidity in preterm neonates. Cochrane Database Syst. Rev. 2019, 11, CD013145. [CrossRef] [PubMed]

71. Grace, E.; Hilditch, C.; Gomersall, J.; Collins, C.T.; Rumbold, A.; Keir, A.K. Safety and efficacy of human milk-based fortifier in enterally fed preterm and/or low birthweight infants: A systematic review and meta-analysis. Arch. Dis. Child. Fetal Neonatal Ed. 2021, 106, 137-142. [CrossRef] [PubMed]

72. John, A.; Sun, R.; Maillart, L.; Schaefer, A.; Spence, E.H.; Perrin, M.T. Macronutrient variability in human milk from donors to a milk bank: Implications for feeding preterm infants. PLoS ONE 2019, 14, e0210610. [CrossRef]

73. Paulaviciene, I.J.; Liubsys, A.; Eidukaite, A.; Molyte, A.; Tamuliene, L.; Usonis, V. The effect of prolonged freezing and holder pasteurization on the macronutrient and bioactive protein compositions of human milk. Breastfeed. Med. 2020, 15, 583-588. [CrossRef] 